\title{
AUTONOMOUS NAVIGATION OF A SPACECRAFT FORMATION IN THE PROXIMITY OF AN ASTEROID
}

\author{
Massimo Vetrisano and Massimiliano Vasile \\ Advanced Space Concepts Laboratory, Department of Mechanical and Aerospace \\ Engineering, University of Strathclyde, James Weir Building, 75 Montrose Street, G1 1XQ \\ Glasgow, UK; $t:+441415482326 f:+441415525105$. \\ \{massimo.vetrisano\}\{massimiliano.vasile\}@strath.ac.uk
}

\begin{abstract}
This paper presents a multi-sensor navigation approach to allow a formation of spacecraft to autonomously navigate in the proximity of a Near Earth Asteroid. Multiple measurements collected by on-board cameras, attitude sensors and LIDAR are used to estimate the state of each spacecraft with respect to the asteroid. Inter-spacecraft position measurements are then combined with spacecraft-to-asteroid position measurements to improve accuracy. The paper analyses the use of different filtering techniques to estimate the state of a 4-spacecraft formation with respect to the asteroid. Different combinations of measurements are constructed to evaluate the improvement in navigation performance offered by the data fusion of the measurements gathered by the four spacecraft. Moreover the robustness of the navigation system is tested against the occurrence of failures. Results show that the navigation performance is significantly improved by adding the inter-spacecraft position measurements. Finally, an asteroid orbit determination method is proposed that combines asteroid's line of sight measurements from multiple spacecraft and Sun Doppler shift sensor with spacecraft-to-ground tracking data. Different approach configurations are evaluated for a 2-spacecraft formation and it is shown that the integrated use of spacecraft-to-asteroid and ground-to-spacecraft measurements provides an effective way to improve the ephemerides of the asteroid.
\end{abstract}

Keywords: multi-sensor navigation, spacecraft formation, inter-spacecraft, unscented Kalman filter, asteroid orbit determination

\section{Introduction}

Near Earth Objects (NEO), the majority of which are asteroids, are defined as any minor celestial object with a perihelion less than 1.3 AU and an aphelion greater than $0.983 \mathrm{AU}$. A subclass of these, deemed potentially hazardous asteroids (PHA), are defined as those with a Minimum Orbital Intersection Distance (MOID) from the Earth's orbit less than or equal to 0.05 AU and a effective diameter larger than $150 \mathrm{~m}$. As of the 11th of June 2015, 12800 NEOs have been detected; of those, more than 3300 have a diameter between 0.3 and $1 \mathrm{~km}$, and 1593 are listed as PHA $^{*}$. Impacts from asteroids of about $1 \mathrm{~km}$ or more in diameter are considered to be capable of causing global climate change and the destruction of ozone, with a land destruction area equivalent to a large state or country. Those with an average diameter of $100 \mathrm{~m}$ can cause significant tsunamis and/or the land destruction of a large city. It is estimated that there are between 30000-300000 NEOs with diameters around $100 \mathrm{~m}$, meaning a large number of NEOs are still undetected.

Different methods have been proposed and studied to deflect PHAs (Melosh et al. 1994, Colombo et al. 2006). Some of them envisage flying a formation (or swarm) of spacecraft in the

\footnotetext{
* Source: http://neo.jpl.nasa.gov/stats
} 
proximity of the asteroid. Maddock et al. (2007) first proposed a formation of solar concentrators, which was extended by Vasile and Maddock (2012) to a swarm of solar-pumped laser systems. Other concepts propose the use of multi-gravity tractors, multi-solar beaming formations (Wie 2008) or the use of a swarm of spacecraft to explore the asteroid belt (Curtis et al. 2003).

This kind of mission requires the spacecraft to fly in a tight formation relatively close to the asteroid. The navigation in close proximity of asteroids can be complicated due to the fact that the environment is uncertain especially if the asteroid presents an irregular shape and is rotating. The motion of the spacecraft around the asteroid is, thus, highly nonlinear. Furthermore, solar radiation pressure can be sufficiently high to strip a spacecraft out of its formation orbit. Generally the gravitational harmonics of the celestial minor bodies are estimated from on-board data collected during a close fly-by (Morley and Budnik 2009), during approach phases (Scheeres 2004a) or by ground-based radar imaging data (Scheeres et al. 2004b). The maintenance of a tight formation around the asteroid is complicated by the fact that the knowledge of these figures is affected by uncertainties due to the relatively limited available information. Although a formation requires controlling an increased number of degrees of freedom, it also provides higher flexibility to achieve mission's goal, can improve overall system's performance and enhance the reliability and robustness of the mission. Thus, it is necessary to define a particular navigation strategy to increase the mission reliability and the possibility to cope with both unknown environment and system performance uncertainties.

Methods based on optical navigation camera and laser light radar (LIDAR) or laser range finder (LRF) integrated measurements has been proved to be a feasible option for a single spacecraft to approach or land on an asteroid (Kubota et al. 2003, Li et al. 2006). Specific work on close proximity operations of a single spacecraft near an asteroid can be found in Broschart and Scheeres (2005) and more recently in Winkler et al. (2013) and Turconi et al. (2014). Previous work in the literature has shown that for multiple spacecraft systems, the inclusion of inter-spacecraft relative observations can improve the estimation of the state of the member of the formation (Long et al. 2002, Shim et al. 2009, and Chung et al. 2006). This paper investigates the possibility to data fuse the measurements of four spacecraft, including inter-satellite links, to improve the navigation performance and reliability of a formation in the proximity of an asteroid. The estimated state of each spacecraft is then fed into a Lyapunov controller to maintain each member of the formation in the desired configuration. This work extends a previous investigation by the authors (Vetrisano and Vasile 2012) in which the idea of navigating a formation of spacecraft in the proximity of an asteroid with a distributed navigation was introduced. The work on the control of formations in the proximity of asteroids up has, up to now, been limited to the control of a formation of solar sail tractors in the proximity of an asteroid (Gong et al. 2009).

It is shown that sharing the information within the member of the formation improves navigation accuracy and allows the formation to be controlled even in the event of contingencies. Furthermore, the paper investigates the possibility of using a 2-spacecraft formation as in-orbit observatory to improve the asteroid's trajectory estimate prior to starting the acquisition of the proximity orbit. On-board optical measurements from single navigation cameras have been widely used in recent years during deep space navigation and approach (Konimato et al. 2006). For instance, relying only on a single camera, the Hayabusa mission performed a set of dogleg manoeuvres to observe the asteroid from different angles to refine the knowledge of the ephemerides of the asteroid. This paper proposes an alternative strategy using more than one spacecraft. It is shown, for different approach configurations, that a 2-spacecraft formation can improve the accuracy of the asteroid ephemerides without performing dogleg maneuvers and 
using instead a combination of in-space measurements and ground based tracking. A similar idea considering only a single spacecraft was discussed in Scheeres (1998).

This paper is organised as follows: the dynamic model of the spacecraft formation is introduced in Section 2 along with a Lyapunov control law designed to maintain the trajectory of each spacecraft close to the reference unperturbed formation orbit. Section 3 presents the measurement models. In Section 4, the filtering process is described along with a data fusion process to deal with data from different spacecraft. Section 6 compares the collaborative to the non-collaborative navigation cases and analyses some contingencies cases. Finally some tests for 2-spacecraft formations are performed to prove that combination of on-board and ground-based measurements can be used to improve the ephemerides of the asteroid alternatively to the dogleg approach.

\section{Dynamic Models}

This section presents the dynamic models that are used in the simulation of the navigation and control of the formation in the proximity of the asteroid, the simulation of the motion of the asteroid and the two state estimation processes to reconstruct the motion of the spacecraft and to improve the ephemerides of the asteroid.

\subsection{Formation Dynamics}

With reference to Figure 1, two coordinate systems are defined to describe the motion of the formation and asteroid:

a) Inertial heliocentric reference frame $S\{\mathbf{I}, \mathbf{J}, \mathbf{K}\}$ (Cui et al. 2010): the Sun is at the centre of the reference frame, the $I$ axis points to the Vernal equinox, the $J$ axis is perpendicular to the $I$ axis in the fundamental plane and the $K$ axis is perpendicular to the fundamental plane pointing towards the north pole of the Sun.

b) Asteroid Hill rotating reference frame $A\left\{\hat{\mathbf{x}}_{h}, \hat{\mathbf{y}}_{h}, \hat{\mathbf{z}}_{h}\right\}:$ the centre $A$ is at the barycentre of the asteroid, the $x$ axis is inwards along the orbit radius, the $y$ axis is perpendicular to the $x$ axis in the orbital plane and the $z$ axis completes the right-handed coordinate system. The relative position $\delta \mathbf{r}^{h}=\left[x_{h}, y_{h}, z_{h}\right]^{T}$ and velocity $\delta \dot{r}^{h}=\left[\dot{x}_{h}, \dot{y}_{h}, \dot{z}_{h}\right]^{T}$ of the spacecraft with respect to the asteroid are described in this coordinate system. 


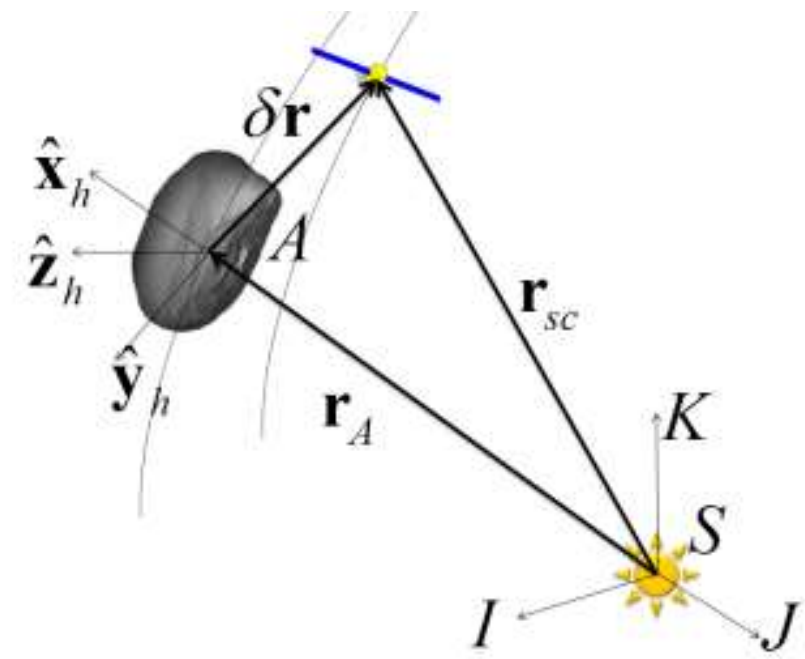

Figure 1. Definition of the reference coordinate systems.

It is assumed that the asteroid is an ellipsoid with semi-axes $a_{I}, b_{I}$ and $c_{I}$ (Scheeres 2010). The asteroid rotates around the $z$-axis with angular velocity $\omega_{A}$ as in Figure 2.

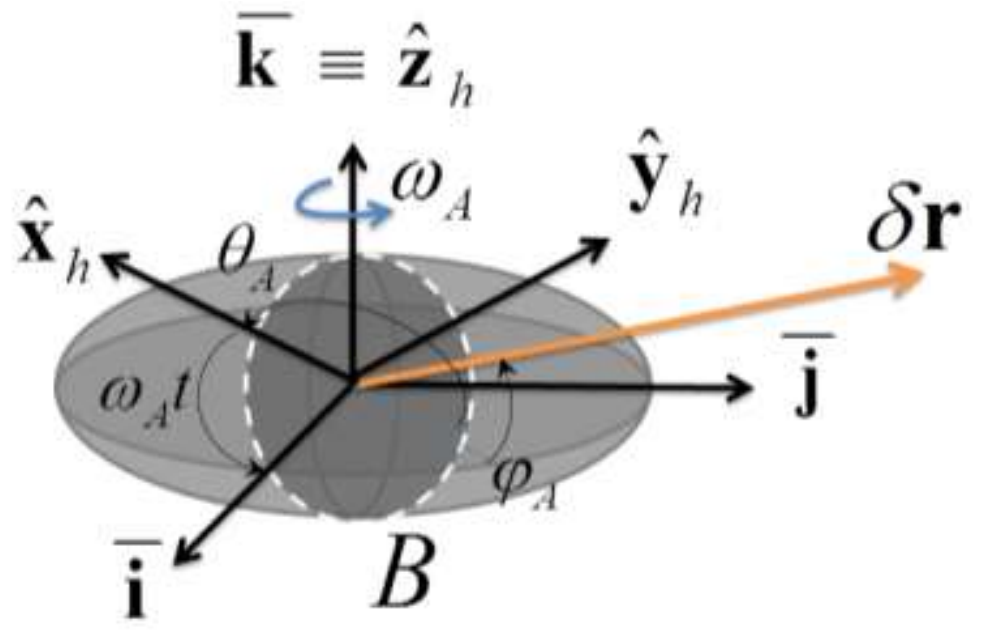

Figure 2. Asteroid body frame with respect to the Hill frame.

The geometric shape model of the asteroid is expressed as

$$
\left(\frac{x_{b}}{a_{l}}\right)^{2}+\left(\frac{y_{b}}{b_{l}}\right)^{2}+\left(\frac{z_{b}}{c_{l}}\right)^{2}=1
$$

where the subscript $b$ refers to the body frame $B\{\overline{\mathbf{i}}, \overline{\mathbf{j}}, \overline{\mathbf{k}}\}$. It has been demonstrated that the seconddegree and second-order gravity field terms dominate the orbital stability of the spacecraft for close proximity motion to uniformly rotating asteroid (Vasile and Maddock, 2012). Therefore, the gravity field of the asteroid can be expressed as the sum of a spherical field plus a second-degree and second-order field (Hu and Scheeres, 2012):

$$
U_{20+22}=\frac{\mu_{A}}{\delta r^{3}}\left(C_{20}\left(1-\frac{3}{2} \cos ^{2} \theta_{A}\right)+3 C_{22} \cos ^{2} \theta_{A} \cos 2 \varphi_{A}\right)
$$


where $\mu_{A}$ is the asteroid gravitational constant, the harmonic coefficients $\mathrm{C}_{20}$ and $\mathrm{C}_{22}$ are a function of the semi-axes:

$$
\begin{aligned}
& C_{20}=-\frac{1}{10}\left(2 c_{l}^{2}-a_{l}^{2}-b_{l}^{2}\right) \\
& C_{22}=\frac{1}{20}\left(a_{l}^{2}-b_{l}^{2}\right)
\end{aligned}
$$

and $\theta_{A}$ and $\varphi_{A}$ are the latitude and longitude angles, respectively, as shown in Figure 2. These two angles are defined as:

$$
\begin{gathered}
\theta_{A}=\arctan \left(\frac{z_{h}}{\sqrt{x_{h}^{2}+y_{h}^{2}}}\right) \\
\varphi_{A}=\arctan \left(\frac{y_{h}}{x_{h}}\right)+\omega_{A} t
\end{gathered}
$$

with $t$ being the time. It is assumed that the asteroid body frame is coincident with the asteroid Hill frame at the beginning of the simulations.

The spacecraft is assumed to be subject to the gravitational force of the Sun, solar radiation pressure and the irregular gravity of the asteroid. The nonlinear relative equations of motion are given by (Vasile and Maddock 2012):

$$
\begin{aligned}
& \ddot{x}_{h}=2 \dot{\theta}\left(\dot{y}_{h}-y_{h} \frac{\dot{r}_{A}}{r_{A}}\right)+x_{h} \dot{\theta}^{2}+\mu_{\text {sun }}\left(\frac{1}{r_{A}^{2}}-\frac{r_{A}+x_{h}}{r_{s c}^{3}}\right)+a_{\text {sun }}-\mu_{A} \frac{x_{h}}{\delta r^{3}}+\frac{\partial U_{20+22}}{\partial x_{h}}+u_{x} \\
& \ddot{y}_{h}=-2 \dot{\theta}\left(\dot{x}_{h}-x_{h} \frac{\dot{r}_{A}}{r_{A}}\right)+y_{h} \dot{\theta}^{2}-\mu_{\text {sun }} \frac{y_{h}}{r_{s c}^{3}}-\mu_{A} \frac{y_{h}}{\delta r^{3}}+\frac{\partial U_{20+22}}{\partial y_{h}}+u_{y} \\
& \ddot{z}_{h}=-\mu_{\text {sun }} \frac{z_{h}}{r_{s c}^{3}}-\mu_{A} \frac{z_{h}}{\delta r^{3}}+\frac{\partial U_{20+22}}{\partial z_{h}}+u_{z}
\end{aligned}
$$

with $\theta$ the true anomaly of the asteroid, $\mu_{\text {Sun }}$ the Sun gravity constant, $\mu_{A}$ the gravity constant of the asteroid, and $r_{A}$ and $r_{s c}$ the orbit radius of the asteroid and spacecraft respectively. The quantity $\dot{r}_{A}$ is the radial velocity of the asteroid and $\delta r$ is the relative distance between the spacecraft and the asteroid. The vector $\mathbf{u}=\left[\begin{array}{lll}u_{x} & u_{y} & u_{z}\end{array}\right]^{T}$ is a control input, which will be defined later in Section 4.

Beyond the gravitational perturbations from the asteroids, the major perturbation acting on the spacecraft is the solar radiation pressure $a_{\text {sun }}$ defined as:

$$
a_{\text {sun }}=C_{R} S_{\text {srp }}\left(\frac{r_{1 A U}}{r_{s C-i}}\right)^{2} \frac{A}{m_{s c}}
$$

where $r_{s c-i}$ is the distance of the $i^{\text {th }}$ spacecraft from the Sun, $A$ and $m_{s c}$ are the spacecraft cross sectional area and mass, respectively, $C_{R}$ is the reflectivity coefficient, $S_{s r p}$ is the solar radiation pressure at $1 \mathrm{AU}$ and $r_{1 A U}$ is one astronomical unit in $\mathrm{km}$. The assumption here is that the components of the radiation pressure not along the radial direction are small and can be seen as a process noise. Note that no spacecraft tugging is considered. For the case in which the spacecraft generates a variation of the orbit of the asteroid please refer to Vetrisano et al. (2013). 
If one considers a formation of 4 spacecraft, the system of Eqs. (6) can be applied to each spacecraft independently and can be re-written in compact form as a system of first order differential equations:

$$
\dot{\mathbf{x}}^{h}=f\left(\mathbf{x}^{h}, \mathbf{u}\right)+\mathbf{w}
$$

where $\mathbf{x}^{h}=\left[\delta \mathbf{r}_{S C-1}^{h}, \delta \dot{\mathbf{r}}_{S C-1}^{h}, \delta \mathbf{r}_{S C-2}^{h}, \delta \dot{\mathbf{r}}_{S C-2}^{h}, \delta \mathbf{r}_{S C-3}^{h}, \delta \dot{\mathbf{r}}_{S C-3}^{h}, \delta \mathbf{r}_{S C-4}^{h}, \delta \dot{\mathbf{r}}_{S C-4}^{h}\right]^{T}$ is the state vector containing the relative position and velocity of all the spacecraft. The vectors $\delta \mathbf{r}_{S C-i}^{h}=\left[x_{i}, y_{i}, z_{i}\right]^{T}$ and $\delta \dot{r}_{s C-i}^{h}=\left[\dot{x}_{i}, \dot{y}_{i}, \dot{z}_{i}\right]^{T}$ are, respectively, the relative position and velocity of the $i^{\text {th }}$ spacecraft with respect to the asteroid, and $\mathbf{w}=\left[0, \mathbf{w}_{s C-1}, 0, \mathbf{w}_{s C-2}, 0, \mathbf{w}_{s C-3}, 0, \mathbf{w}_{S C-4}\right]^{T}$ is an additional vector modelling the process noise. The magnitude of this noise is set to $10^{-9} \mathrm{~m} / \mathrm{s}^{2}$ for all spacecraft.

The spacecraft formation is assumed to fly in the proximity of the NEO 99942 Apophis as a representative example of medium size non-spherical S-class asteroid. The Keplerian elements along with the physical properties of Apophis are listed in Table 1.

The initial position and velocity of each spacecraft with respect to the asteroid in the Hill's reference frame are given in Table 2. If the gravity of the asteroid and radiation pressure are neglected, the initial conditions in Table 2 provide the periodic formation orbits in Figure 3 . In the following, these formation orbits will be taken as reference nominal trajectories for the four spacecraft. The initial mass of each spacecraft is $500 \mathrm{~kg}$, and the maximum cross section area is $20 \mathrm{~m}^{2}$. A mean value of 1.2 for the reflectivity coefficient is assumed, with a uniformly distributed uncertainty of $20 \%$ on the resulting value of the solar radiation pressure.

Table 1. Orbital and physical properties of 99942 Apophis.

\begin{tabular}{ccc}
\hline Element & Notation & Value \\
\hline Semi-major axis & $a_{A}$ & $0.9224 \mathrm{AU}$ \\
Eccentricity & $e_{A}$ & 0.1912 \\
Inclination & $i_{A}$ & $0.05814 \mathrm{rad}$ \\
Longitude of ascending note & $\Omega_{A}$ & $3.05682 \mathrm{rad}$ \\
Argument of periapsis & $\varpi_{A}$ & $2.2061 \mathrm{rad}$ \\
Orbital Period & $T_{A}$ & $323.5969 \mathrm{~d}$ \\
Gravitational constant & $\mu_{A}$ & $1.801599 \times 10^{-9} \mathrm{~km}^{3} / \mathrm{s}^{2}$ \\
Physical dimensions & $a_{l}, b_{l}, c_{l}$ & $191 \mathrm{~m}, 135 \mathrm{~m}, 95 \mathrm{~m}$ \\
Rotational velocity & $\omega_{A}$ & $5.8177 \times 10^{-5} \mathrm{rad} / \mathrm{s}$ \\
Absolute magnitude & $M$ & 19.7 \\
\hline
\end{tabular}

Table 2. Initial spacecraft trajectory parameters.

\begin{tabular}{lclcccc}
\hline & $x_{h}(\mathrm{~km})$ & $y_{h}(\mathrm{~km})$ & $z_{h}(\mathrm{~km})$ & $\dot{x}_{h}\left(10^{-9} \mathrm{~km} / \mathrm{s}\right)$ & $\dot{y}_{h}\left(10^{-9} \mathrm{~km} / \mathrm{s}\right)$ & $\dot{z}_{h}\left(10^{-9} \mathrm{~km} / \mathrm{s}\right)$ \\
\hline SC1 & 0.0323 & -0.5000 & -0.774 & 0.193 & -4.480 & -7.837 \\
SC2 & 0.046 & -1.039 & -0.608 & 0.051 & -18.120 & -6.350 \\
SC3 & 0.0323 & -0.503 & 0.307 & 0.259 & -4.533 & -3.652 \\
SC4 & 0.092 & -1.104 & 0.451 & 0.009 & -1.467 & -4.942 \\
\hline
\end{tabular}




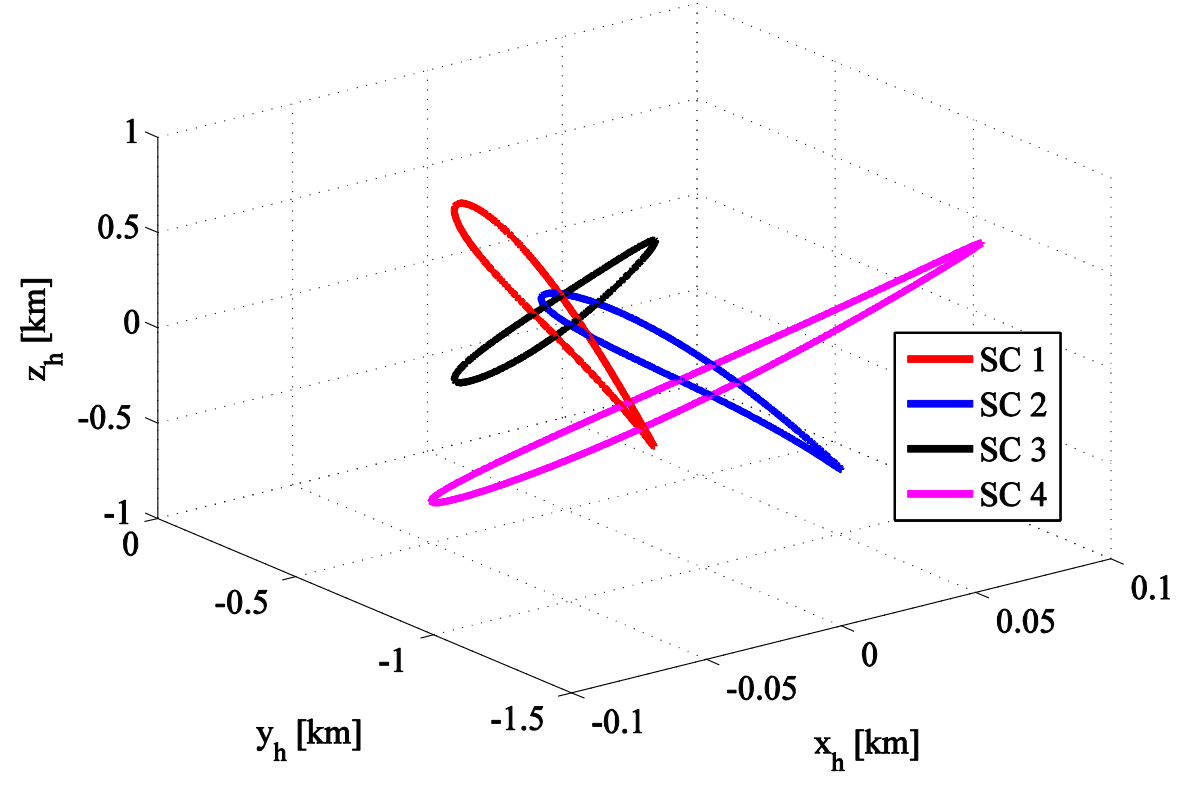

Figure 3. Nominal trajectories.

\subsection{Dynamics of Asteroid and Spacecraft in the Heliocentric Frame}

Section 6.3 will present an approach to improve the ephemerides of the asteroid using two spacecraft flying in formation. The orbit determination process employs the dynamic equations of the asteroids in an inertial Sun-centred reference frame written as:

$$
\begin{aligned}
& \dot{\mathbf{r}}_{A}=\mathbf{v}_{A} \\
& \ddot{\mathbf{r}}_{A}=-\frac{\mu_{\text {Sun }}}{r_{A}^{3}} \mathbf{r}_{A}+\mathbf{w}_{A}
\end{aligned}
$$

where $\mathbf{v}_{A}$ is the asteroid velocity in the heliocentric frame, and $\mathbf{w}_{A}$ is a Gaussian white noise vector with uncorrelated components. Note that the perturbations acting on the asteroid are assumed to be null and no effects such as solar radiation were included. The dynamics of the spacecraft, far from the asteroid, are defined in an inertial Sun-centre reference frame and governed by the following system of differential equations that includes a noise term $\mathbf{w}_{s c-i}$ to represent model uncertainty:

$$
\dot{\mathbf{x}}_{s C-i}=f_{H}\left(\mathbf{x}_{s C-i}\right)=\left[\begin{array}{c}
\dot{r}_{s C-i} \\
-\frac{\mu_{S u n}}{r_{S C-i}^{3}} \mathbf{r}_{s c-i}+C_{R} S_{s r p}\left(\frac{r_{1 A U}}{r_{s c-i}}\right)^{2} \frac{\mathbf{r}_{s C-i}}{r_{s C-i}} \frac{A}{m_{s c}}+\mathbf{w}_{s c-i}
\end{array}\right]
$$

where $\mathbf{x}_{s c-i}=\left[\mathbf{r}_{s C-i}, \dot{r}_{s C-i}\right]^{T}$ is the spacecraft state in the heliocentric reference frame. Note that the hypothesis here is that light pressure and Yarkovsky effect are to be considered secondary perturbations to the motion of the asteroid while light pressure is an important perturbation component for the motion of the spacecraft. 


\section{Measurements Model}

With reference to Figure 4, it is assumed that each spacecraft is provided with the following set of sensors and measurements:

a) A high resolution camera which provides elevation and azimuth angles of feature points on the surface of the asteroid.

b) A LIDAR which measures the distance from the spacecraft to a point on the asteroid's surface.

c) Inter-spacecraft measurements, which include the relative attitude and the distance between two spacecraft.

d) Range and range rate obtained from ground stations.

e) Sun Doppler shift sensor measuring the radial velocity of the spacecraft with respect to the Sun.

Note that other combinations are possible, for example one could exploit the formation to acquire distance measurements only with the camera. However, the intent here is to improve the navigation performance of a single spacecraft and to test the ability of the formation to operate correctly by data fusing inhomogeneous measurements when the measurements are degraded. For this reason each spacecraft is equipped with a set of instruments that would be sufficient to navigate in the proximity of the asteroid. The model of each of these measurements is presented in the following subsections.
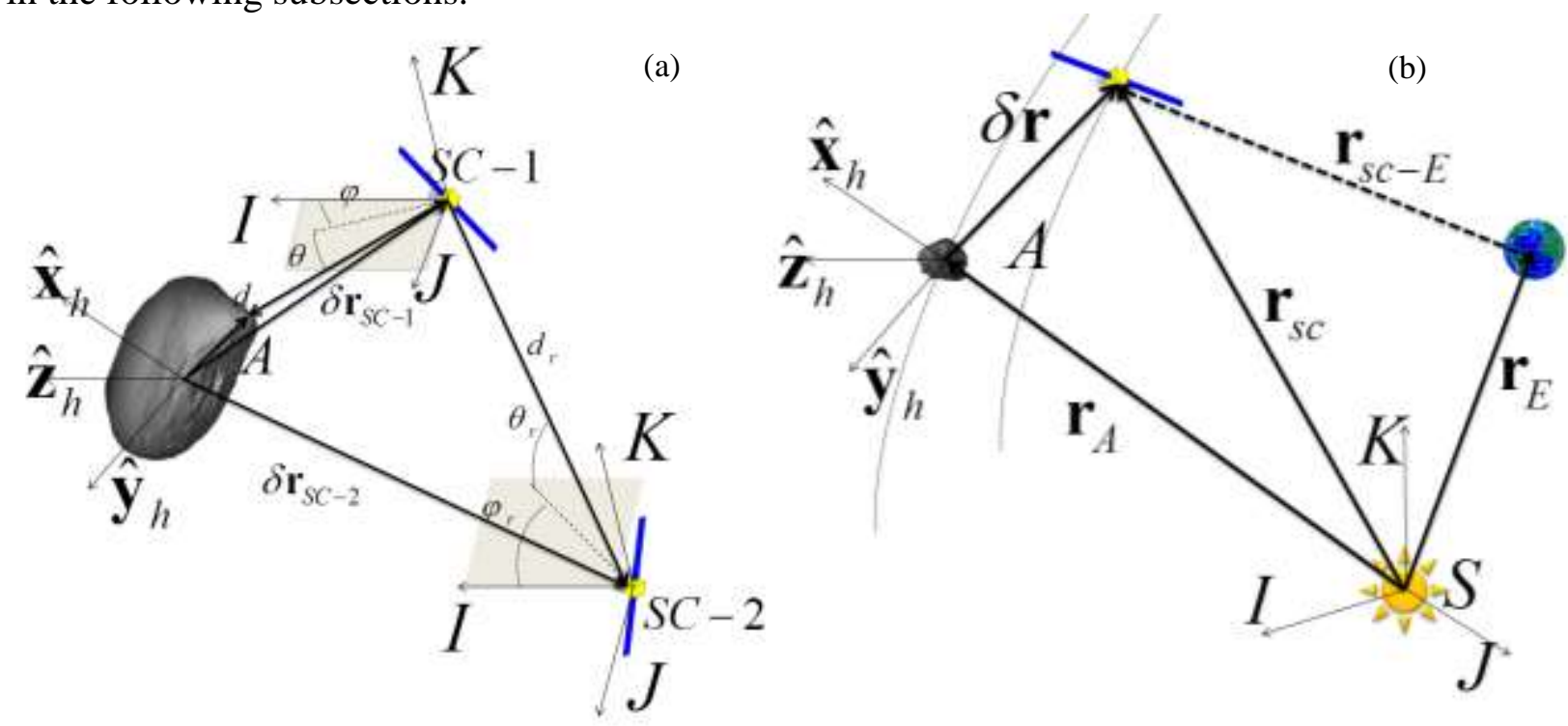

Figure 4. Measurements models for (a) relative navigation geometry, (b) absolute navigation geometry.

\subsection{Camera Model}

In order to develop the measurement model of the camera, two intermediate reference frames are required as shown in Figure 5:

1) Spacecraft coordinate system $S C\left\{\mathbf{x}_{s c}, \mathbf{y}_{s c}, \mathbf{z}_{s c}\right\}$ (Li et al. 2007): the origin of this frame lies on the centre of mass of the spacecraft, with the three symmetrical body axes defined as three coordinate axes.

2) Camera coordinate system $C\left\{\hat{\mathbf{x}}_{c}, \hat{\mathbf{y}}_{c}, \hat{\mathbf{z}}_{c}\right\}$ : the centre $C$ is the perspective projection of the camera, with the $x_{c}$-axis parallel to the optical axis of the camera and directed to the centre of the 
asteroid. The image plane is defined as $o_{c}-z_{c} y_{c}$. To simplify mathematics, it is assumed that the spacecraft and camera coordinate systems are coincident.

In the following, for sake of simplicity, during the proximity phases the spacecraft coordinate system is assumed to be aligned with the Hill frame $A$ (see Section 2), while during the deep space navigation phase the coordinate system is aligned with the inertial frame $\mathrm{S}$. Thus, it is assumed that the attitude of each spacecraft is known with a level of precision corresponding to the one of the star trackers on two axes.

Having identified the two reference frames, one can define the geometric relationship in the asteroid Hill rotating reference frame. The position vector of the $i^{\text {th }}$ feature is $\mathbf{x}_{\text {surface }}^{i}$, which is selected randomly on the asteroid surface defined in Eq. (1). The spacecraft position vector with respect to the asteroid is defined as $\delta \mathbf{r}_{s c}$, while $\mathbf{x}_{\text {surf-sc }}^{i}$ refers to the position vector from the estimated spacecraft to the feature.

With reference to Figure 5, assuming a pinhole model for the camera (Oh and Johnson 2007), a point on the surface of the asteroid with position $r_{p}=\left[\begin{array}{lll}x_{c} & y_{c} & z_{c}\end{array}\right]^{T}$ in the reference frame of the camera has coordinates on the image plane given by:

$$
\left[\begin{array}{l}
u \\
v
\end{array}\right]=\frac{f}{x_{c}}\left[\begin{array}{l}
y_{c} \\
z_{c}
\end{array}\right]
$$

where $x_{c}$ is the distance of the point from the image plane along the boresight direction and $f$ is the focal length of the camera.

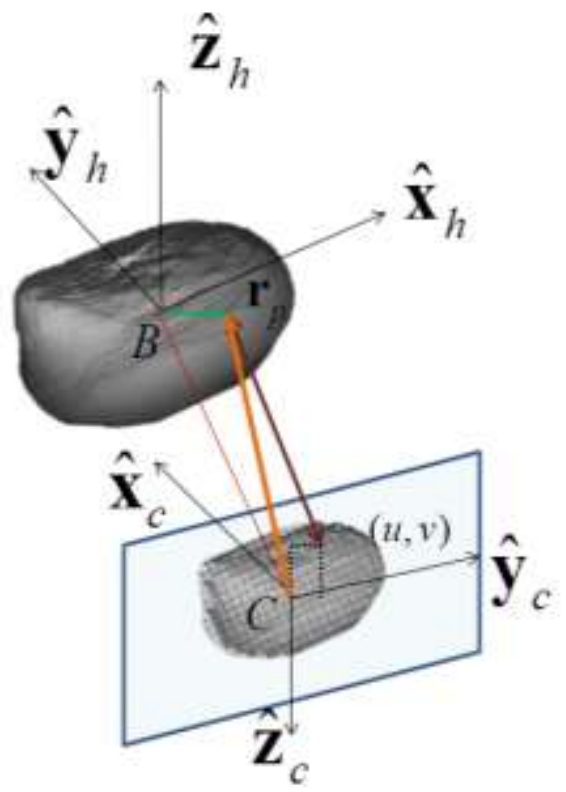

Figure 5. Pin-Hole camera model.

Without taking into account the effect of the attitude error, the position in the camera reference frame would be given by:

$$
\mathbf{r}_{p}=\mathbf{R}_{H C} \mathbf{x}_{\text {Surf }-s C}
$$

where $\mathbf{R}_{H C}$ is the rotation matrix from the Hill's reference frame to the camera frame and:

$$
\mathbf{x}_{\text {surf-sC }}=\mathbf{x}_{\text {surface }}-\delta \mathbf{r}_{s C}
$$


with $\mathbf{x}_{\text {surface }}$ the vector position of the points with respect to the centre of the Hill's reference frame. The coordinates of the point on the image plane measured in pixels are given by:

$$
\begin{aligned}
& x_{\text {screen }}=u / p_{\text {width }} \\
& y_{\text {screen }}=v / p_{\text {width }}
\end{aligned}
$$

with $p_{\text {width }}$ the pixel width. If one considers the effect of the attitude errors $\lambda_{1,2}$ around $\hat{\mathbf{z}}_{c}$ and $\hat{\mathbf{y}}_{c}$, the vector $\mathbf{r}_{p}$ of each feature will be subject to a random rotation in the reference frame of the camera given by:

$$
\mathbf{r}_{p}^{a}=\mathbf{R}_{\text {attitude }} \mathbf{r}_{p}=\left[\begin{array}{ccc}
\cos \lambda_{1} \cos \lambda_{2} & \sin \lambda_{1} & \cos \lambda_{1} \\
-\sin \lambda_{1} \cos \lambda_{2} & \cos \lambda_{1} & \sin \lambda_{2} \\
-\sin \lambda_{2} & 0 & \cos \lambda_{2}
\end{array}\right]\left[\begin{array}{c}
x_{c} \\
y_{c} \\
z_{c}
\end{array}\right]
$$

In the case of small errors in the pointing angles, substituting $\mathbf{r}_{p}^{a}$ into Eq. (11) gives:

$$
\left[\begin{array}{l}
u \\
v
\end{array}\right]=\frac{f}{x_{c}+\lambda_{1} y_{c}+\lambda_{2} z_{c}}\left[\begin{array}{l}
y_{c}-\lambda_{1} x_{c} \\
z_{c}-\lambda_{2} x_{c}
\end{array}\right]
$$

where the attitude error contribution increases with the distance. Neglecting the terms in the denominator that are multiplied by a pointing angle, Eq. (16) leads to:

$$
\left[\begin{array}{l}
u \\
v
\end{array}\right]=\frac{f}{x_{c}}\left[\begin{array}{l}
y_{c} \\
z_{c}
\end{array}\right]+\left[\begin{array}{l}
-\lambda_{1} f \\
-\lambda_{2} f
\end{array}\right]
$$

The mean position of all the points on the image plane of the camera defines the coordinates of the centroid of the asteroid $\left({ }^{c} x_{c},{ }^{c} y_{c}\right)=\left({ }^{c} u_{c},{ }^{c} v_{c}\right) p_{\text {width }}$. By then measuring the angular position of the centroid, one can estimate the angular position of the centre of mass in the reference frame of the camera. The azimuth and elevation angles of the centroid are given by:

$$
\begin{aligned}
& \varphi=\tan ^{-1} \frac{{ }^{c} x_{c}}{f} \\
& \psi=\tan ^{-1} \frac{{ }^{c} y_{c}}{\sqrt{{ }^{c} x_{c}^{2}+f^{2}}}
\end{aligned}
$$

It is assumed that the centroid of the asteroid identifies the position of the centre of mass with some uncertainty. The measurement from the camera is affected by the spacecraft attitude pointing, the pixelization and the centroiding errors (where the last one is the mismatch between centroid and centre of mass). The pixelization error is due to the fact that the image of the asteroid is formed by a discrete number of pixels. By expressing Eq. (18) as a function of the pixel size and coordinates on the image plane, one can write the observation equation:

$$
\mathbf{z}_{\text {camera }}=\left[\begin{array}{c}
\tan ^{-1} \frac{{ }^{c} u_{c} p_{\text {width }}+\varsigma_{p}+\varsigma_{\varphi}^{A}}{f} \\
\tan ^{-1} \frac{{ }^{c} v_{c} p_{\text {width }}+\varsigma_{p}+\varsigma_{\psi}^{A}}{\sqrt{\left({ }^{c} u_{c} p_{\text {width }}+\varsigma_{p}+\zeta_{\varphi}^{A}\right)^{2}+f^{2}}}
\end{array}\right]
$$


where $\varsigma_{p}$ is the pixelization error, and $\varsigma_{\varphi}^{A}$ and $\varsigma_{\psi}^{A}$ are the attitude and centroiding errors. With the simplifications of Eq. (17), the latter terms would be:

$$
\left[\begin{array}{c}
\varsigma_{\varphi}^{A} \\
\varsigma_{\psi}^{A}
\end{array}\right]=-\left[\begin{array}{l}
p_{\text {width }} \lambda_{1} f \\
p_{\text {width }} \lambda_{2} f
\end{array}\right]
$$

Expanding Eq. (19) up to the first order in the noise component, one obtains:

$\mathbf{z}_{\text {camera }}=\left[\begin{array}{c}\tan ^{-1} \frac{{ }^{c} u_{c} p_{\text {width }}}{f} \\ \tan ^{-1} \frac{{ }^{c} v_{c} p_{\text {width }}}{\sqrt{\left({ }^{c} u_{c} p_{\text {width }}\right)^{2}+f^{2}}}\end{array}\right]+\ldots$

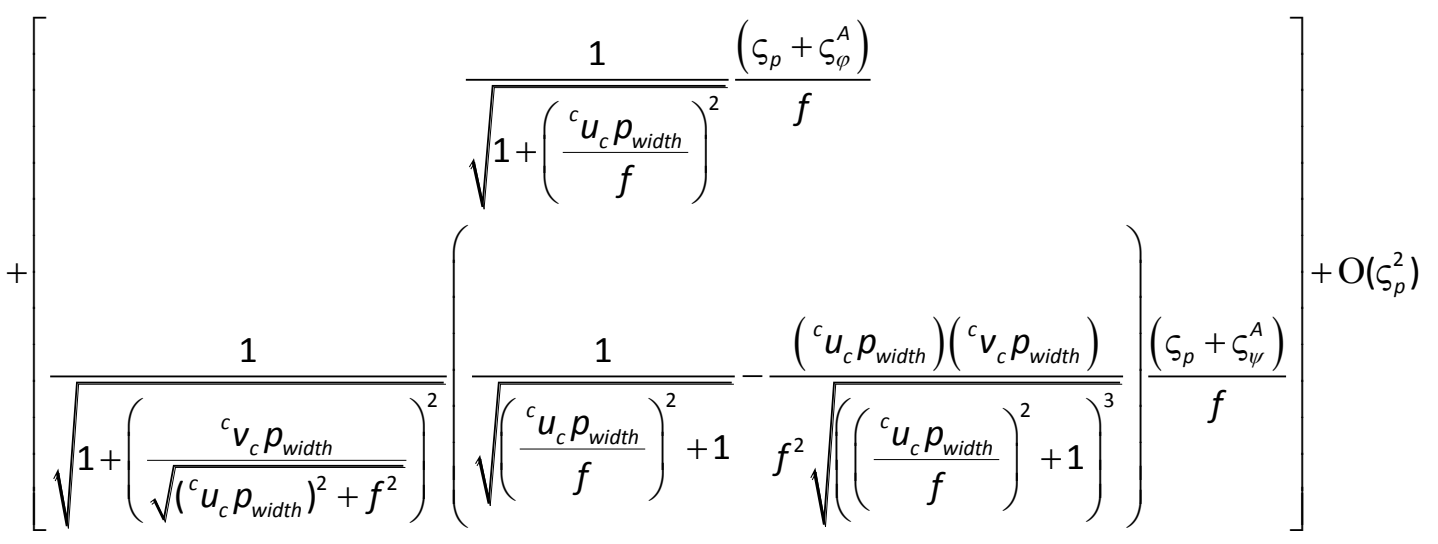

From Eq. (21), one can see that the worst case error is achieved when the point is located at the centre of the screen. This means that in the worst case

$$
\mathbf{z}_{\text {camera }} \leq\left[\begin{array}{c}
\tan ^{-1} \frac{{ }^{c} u_{c} p_{\text {width }}}{f} \\
\tan ^{-1} \frac{{ }^{c} v_{c} p_{\text {width }}}{\sqrt{\left({ }^{c} u_{c} p_{\text {width }}\right)^{2}+f^{2}}}
\end{array}\right]+\left[\begin{array}{c}
\frac{\varsigma_{p}+\varsigma_{\varphi}^{A}}{f} \\
\frac{\varsigma_{p}+\varsigma_{\psi}^{A}}{f}
\end{array}\right]
$$

The model for the observation equations used in the filter, neglecting the contribution given by the attitude system, becomes:

$$
\mathbf{z}_{\text {camera }}=h_{\text {camera }}(\delta \mathbf{r}, \mathbf{q})+\boldsymbol{\varsigma}_{\text {camera }}=\left[\begin{array}{c}
\tan ^{-1} \frac{{ }^{c} u_{c} p_{\text {width }}}{f} \\
\tan ^{-1} \frac{{ }^{c} v_{c} p_{\text {width }}}{\sqrt{\left({ }^{c} u_{c} p_{\text {width }}\right)^{2}+f^{2}}}
\end{array}\right]+\left[\begin{array}{l}
S_{\varphi} \\
\varsigma_{\psi}
\end{array}\right]
$$

where $\varsigma_{\varphi, \psi}$ comprises all the errors from attitude and centroiding process. Note that here the illumination conditions are not considered, so it is assumed that each spacecraft sees the whole visible surface from its position. This is sensible if one assumes that a complementary map could be built while starting the orbit acquisition, combining pictures from the whole formation. In the 
absence of a map that relates the centroid to the centre of mass, the navigation system would rely on the centre of brightness which, depending on shape and solar aspect angle, could introduce a bias in the determination of the relative position.

\subsection{LIDAR Model}

In general, the LIDAR provides range from the spacecraft to a point on the surface of target object and works at a range from $50 \mathrm{~m}$ to $50 \mathrm{~km}$ (Kubota et al. 2003). It is assumed that the LIDAR illuminates the point on the surface that corresponds to the centroid derived from the elaboration of the images acquired by the camera (Dionne 2009). This distance is simply given by:

$$
I=\left|\delta \mathbf{r}_{s c}^{h}-\mathbf{x}_{\text {surface }}^{c}\right|
$$

where $\mathbf{x}_{\text {surface }}^{c}$ is the position of a point on the asteroid's surface along the centroid direction. The observation equation of the LIDAR including the measurement noise is:

$$
y_{l}=h_{l}\left(\delta \mathbf{r}_{s c}^{h}\right)+\varsigma_{l}=I+\varsigma_{l}
$$

with $\varsigma_{1}$ the measurement noise. The accuracy of this measurement depends on the characteristic error of the sensor, along with a bias defined by the mounting error of the instrument. If the range $l$ is pre-processed in combination with the angular measurements from Eq. (18), a relative position vector from the spacecraft to the point on the surface can be constructed as

$$
\mathbf{z}=\left[\begin{array}{l}
I \\
\varphi \\
\psi
\end{array}\right]=h\left(\delta \mathbf{r}_{s c}^{h}\right)+\mathbf{s}
$$

where $\mathbf{z}$ is the measurement vector obtained from the combination of camera and LIDAR, $h(\mathbf{x})$ is the vector containing the measurement model and $\mathbf{s}$ is the total measurement noise vector. It is important to remark that in the simulations, the errors of the two angles are derived from Eqs. (15) and (19), while the observation equations used in the filter are Eq. (23) and (26).

\subsection{Inter-spacecraft Measurements}

The set of inter-spacecraft measurements is represented by the relative position vector between two spacecraft in the formation. Similarly to the model in Section 3.2, this is composed of the relative distance, local azimuth and elevation (Alonso et al. 2001). The measurements are given by the on-board camera and ranging instruments from one spacecraft to another. For example, if spacecraft 1 measures the relative position of spacecraft 2 , the measurement can be modelled as:

$$
\begin{aligned}
& d_{r e l}=\left|\delta \mathbf{r}_{s c 1}-\delta \mathbf{r}_{s c 2}\right| \\
& \varphi_{r e l}=\tan ^{-1} \frac{y_{12}}{x_{12}} \\
& \theta_{r e l}=\tan ^{-1} \frac{f}{\sqrt{\left(x_{12}\right)^{2}+\left(y_{12}\right)^{2}}}
\end{aligned}
$$


where $d_{r e l}$ is the relative distance between the two spacecraft, $\varphi_{\text {rel }}, \theta_{\text {rel }}$ are, respectively, the local azimuth and elevation angles measured from one spacecraft to the other, and $\left[x_{12}, y_{12}\right]^{T}$ are the coordinates of spacecraft 2 on the screen of the camera of spacecraft 1 . The observation equation is given by:

$$
\mathbf{z}_{r e l}=h_{r e l}\left(\delta \mathbf{r}_{S C 1}^{h}, \delta \mathbf{r}_{S C 2}^{h}\right)+\boldsymbol{\varsigma}_{r e l}=\left[d_{r e l} \varphi_{r e l} \theta_{r e l}\right]^{T}+\boldsymbol{\varsigma}_{r e l}
$$

where $\boldsymbol{\zeta}_{r e l}=\left[\varsigma_{d}, \varsigma_{\theta_{r e l}}, \varsigma_{\varphi_{r e l}}\right]^{T}$ is the measurement noise.

\subsection{Ground Station Measurements}

The set of measurements defined by range $\rho$ and range rate $\dot{\rho}$ with respect to the ground station is the typical set used to estimate a spacecraft trajectory from Earth (Thornton and Border 2003) and is employed during deep space navigation. These values are described in the SouthEast-Zenith reference frame:

$$
\begin{aligned}
& \rho=\left|\boldsymbol{\rho}_{S E Z}\right| \\
& \dot{\rho}=\frac{\dot{\boldsymbol{\rho}}_{S E Z} \cdot \boldsymbol{\rho}_{S E Z}}{\rho}
\end{aligned}
$$

where $\boldsymbol{\rho}_{S E Z}$ and $\dot{\boldsymbol{\rho}}_{S E Z}$ are the position and velocity vectors of the spacecraft measured from ground station. The observation equation is given as

$$
\mathbf{z}_{g}=h_{g}\left(\mathbf{r}_{S C}\right)+\mathbf{u}_{g}=\left[\begin{array}{ll}
\rho & \dot{\rho}
\end{array}\right]^{T}+\mathbf{S}_{g}
$$

with $\varsigma_{g}$ assumed to be the measurement noise.

\subsection{Sun Doppler Shift Sensor Model}

The Doppler shift from sunlight can be measured by using a resonance-scattering spectrometer instrument which allows the measurement of the radial velocity of the spacecraft with respect to the Sun (Yim et al. 2000). This sensor is useful during the deep space navigation phase preasteroid encounter since one can assume that the formation could be not visible from ground for some periods of time. The measurement model of Doppler shift can be modelled by the following equation:

$$
\dot{\rho}_{s c}^{\text {sun }}=\frac{\mathbf{r}_{S C} \cdot \dot{\mathbf{r}}_{S C}}{r_{s c}}=\frac{\mathbf{r}_{S C} \cdot \mathbf{v}_{S C}}{r_{s C}}
$$

The observation equation is given by:

$$
\mathbf{z}_{\text {sun }}=h_{\text {sun }}\left(\mathbf{r}_{s c}\right)+\mathbf{u}_{\text {sun }}=\dot{\rho}_{\text {sc }}^{\text {sun }}+\varsigma_{\text {sun }}
$$

where $\dot{\rho}_{s c}^{\text {sun }}=\dot{\mathbf{r}}_{s c}^{T} \mathbf{r}_{s c} / r_{s c}$ is the relative radial velocity of spacecraft relative to the Sun, and $\varsigma_{s u n}$ is the measurement noise.

\section{Control Strategy}

The control strategy aims at keeping each spacecraft orbiting on a trajectory proximal to the nominal one defined in Figure 3. Note that this is not necessarily an optimal control strategy but 
is sufficient to demonstrate the effect of the navigation and data fusion algorithms. The control law is derived from the following simple Lyapunov control function (Vasile and Maddock 2012):

$$
V=\frac{1}{2} \delta \dot{r}^{2}+\frac{1}{2} \kappa\left(\left(x-x_{\text {ref }}\right)^{2}+\left(y-y_{\text {ref }}\right)^{2}+\left(z-z_{\text {ref }}\right)^{2}\right)
$$

where $\delta \mathbf{r}_{\text {ref }}=\left[\begin{array}{lll}x_{\text {ref }} & y_{\text {ref }} & z_{\text {ref }}\end{array}\right]^{T}$ are the desired coordinates of a spacecraft along the nominal formation orbit in the Hill reference frame, $\delta \mathbf{r}=\left[\begin{array}{lll}x & y & z\end{array}\right]^{T}$ and $\delta \dot{\mathbf{r}}=\left[\begin{array}{lll}\dot{x} & \dot{y} & \dot{z}\end{array}\right]^{T}$ are, respectively, the actual position and velocity of the spacecraft, and $\kappa$ is an elastic coefficient. It is assumed that the motion along the reference formation orbit is much slower than the control action. Furthermore, if one assumes that the only accelerations disrupting the nominal proximity motion are those due to the spherical component of the gravity of the asteroid and to solar radiation pressure, then the controller $\mathbf{u}$ which makes $d V / d t<0$ is defined as follows:

$$
\mathbf{u}=-\left(\mathbf{a}_{\text {sun }}(\delta \mathbf{r})-\frac{\mu_{A}}{\delta r^{3}} \delta \mathbf{r}\right)-\kappa\left(\delta \mathbf{r}-\delta \mathbf{r}_{r e f}\right)-c_{d} \delta \dot{\mathbf{r}}
$$

where $c_{d}$ is a steady dissipation coefficient. If the actual trajectory of the spacecraft is known, the continuous control in Eq. (35) can be introduced into the full dynamic model in Eq. (6). Here however, the trajectory is estimated by the navigation system with the actual position of the spacecraft never known exactly. The predicted estimation is used by the controller to maintain the relative formation (shown later in Section 5). Once the controller is inserted in the spacecraft dynamic model, one obtains a closed loop problem in which the control is performed together with the estimation, and the filter equations incorporate the action of the controller. During the controlled phases, it is assumed that the asteroid trajectory is precisely known; the state variables to be estimated are only those related to the spacecraft in the formation.

\section{State Estimation and Data Fusion Strategy}

A suitable filtering technique needs to be implemented in order to process the measurements defined in Section 3. Given the non-linearities in the dynamics and measurements, a natural choice would be to use a non-linear filtering technique. The work in Vetrisano and Vasile (2012) for collaborative spacecraft shows that the unscented Kalman filter (UKF) is preferable to other non-linear techniques such as the particle filter and the high order expansions filters because it can accurately handle nonlinearities at a low computational cost.

The hypothesis underneath the UKF is that the noise from measurements, dynamic model and priors is Gaussian in nature. This might not be the case in general and even though the UKF has proven to work reasonably well when the Kalman filter hypotheses are not satisfied, a better alternative would be to use an $H_{\infty}$ filter, also called minmax filter. The $H_{\infty}$ filter does not require prior assumptions on the nature of the noise, and minimizes the worst-case estimation error. The choice of the $H_{\infty}$ filter is preferable when the Gaussian hypothesis cannot be fully guaranteed, for example when biases in the instruments are not detected (Simons 2006). In our case, besides biases affecting all the instruments, the LIDAR measurements are affected by the camera process and errors. Therefore, the noise introduced by the LIDAR cannot be modelled as an uncorrelated white noise.

In order to deal with nonlinearities, one can use the Extended $H_{\infty}$ Filter (EHF), analogous to the extended Kalman filter. In this case, however, some hypotheses need to be introduced on the smoothness and regularity of the process and measurements. An alternative is to introduce the 
unscented transformation in the $H_{\infty}$ filter to avoid the approximation of the Jacobian matrices (Li and Jia, 2010) and build an Unscented $H_{\infty}$ Filter (UHF).

The UHF, as well as the UKF, works on the premise that one can find a good approximation for the posteriori covariance by propagating a limited set of optimally chosen samples (Julier et al. 1995). The UHF is hereafter briefly described.

Using the estimation theory formalism, the nonlinear process in Eq. (8) and measurement equations in Section 3 can be discretized in time and written as:

$$
\begin{gathered}
\mathbf{x}_{k+1}=f\left(\mathbf{x}_{k}, \mathbf{u}_{k}, \mathbf{w}_{k}\right) \\
\mathbf{y}_{k}=h\left(\mathbf{x}_{k}, \mathbf{v}_{k}\right)
\end{gathered}
$$

where $\mathbf{w}_{k}$ is the process noise and $\mathbf{v}_{k}$ is the measurement noise. The process noise could belong to a generic distribution but in the following we restrict the analyses to the case in which $\mathbf{w}_{k} \sim N\left(0, \mathbf{Q}_{k}\right)$, with $\mathbf{Q}_{\mathrm{k}}$ the process noise covariance at time step $k$. The quantity $\mathbf{u}_{k}$ represents the control input required to counteract the perturbations acting on the spacecraft (see Section 4$)$. The control is based on the estimated state $\tilde{\mathbf{x}}$, represented by $\delta \tilde{\mathbf{r}}$ and $\delta \tilde{\dot{\mathbf{r}}}$, or the position and velocity of each spacecraft with respect to the asteroid. Thus the controller defined in Eq. (35) becomes:

$$
\mathbf{u}_{k}(\tilde{\mathbf{x}}, t)=-\left(\mathbf{a}_{\text {sun }}\left(\delta \tilde{r}_{k}(t)\right)-\frac{\mu_{A}}{\delta r_{\text {est }}^{3}} \delta \tilde{\mathbf{r}}_{k}(t)\right)-\kappa\left(\delta \tilde{\mathbf{r}}_{k}(t)-\delta \mathbf{r}_{r e f}\right)-c_{d} \delta \tilde{\vec{r}}_{k}(t)
$$

The control input is defined as a function of time to stress that the control is a continuous function between $t_{k}$ and $t_{k+1}$ and is based on the estimated current position and velocity of the spacecraft. The estimated motion $\tilde{\mathbf{r}}_{k}(t)$ and $\delta \tilde{\vec{r}}_{k}(t)$ between $t_{k}$ and $t_{k+1}$ in which the measurements are received and processed, is simply given by the integration of Eq.(8) without the contribution of $\mathbf{w}$. The initial conditions are the estimated position and velocity from the filter at time $t_{k}$. Similarly to the UKF, the UHF relies on the unscented transformation to propagate a set of suitable sigma points, drawn from the apriori covariance matrix. The set of sigma points $\boldsymbol{\chi}_{i}$ are given as:

$$
\boldsymbol{X}_{i}=\left\{\begin{array}{cl}
\tilde{\mathbf{x}}_{k} & i=0 \\
\tilde{\mathbf{x}}_{k}+\left(\sqrt{\left(n+\kappa_{u k f}\right) \mathbf{P}_{k}+\mathbf{Q}_{k}}\right)_{i} & i=1,2, \ldots, n \\
\tilde{\mathbf{x}}_{k}-\left(\sqrt{\left(n+\kappa_{u k f}\right) \mathbf{P}_{k}+\mathbf{Q}_{k}}\right)_{i} & i=n+1, \ldots, 2 n
\end{array}\right.
$$

where $\boldsymbol{x}_{i}$ is a matrix consisting of $(2 n+1)$ vectors with $\kappa_{u k f}=\alpha_{u k f}^{2}\left(n+\lambda_{u k f}\right)-n$, where $\kappa_{u k f}$ is a scaling parameter, and constant $\alpha_{u k f}$ determines the extension of these vectors around $\tilde{\mathbf{x}}_{k}$. We set $\alpha_{u k f}$ equal to $10^{-3}$ and $\lambda_{u k f}$ is set equal to $(3-n)$.

The sigma points are transformed or propagated through the nonlinear function, the so-called unscented transformation, to give:

$$
\begin{aligned}
& \boldsymbol{\chi}_{i, k+1}=f\left(\mathbf{x}_{i, k}, \mathbf{u}_{k}\right) \\
& \mathbf{Y}_{i}=h\left(\mathbf{x}_{i, k}\right)
\end{aligned}, i=0,1, \ldots, 2 n
$$


The mean value and covariance of $\mathbf{y}$ are approximated using the weighted mean and covariance of the transformed vectors (Julier et al. 1995)

$$
\begin{gathered}
\bar{y}=\sum_{i=0}^{2 n} W_{i}^{(m)} \mathbf{Y}_{i} \\
\mathbf{P}_{y}=\sum_{i=0}^{2 n} W_{i}^{(c)}\left(\mathbf{Y}_{i}-\bar{y}\right)\left(\mathbf{Y}_{i}-\bar{y}\right)^{T}
\end{gathered}
$$

where $W_{i}^{(m)}$ and $W_{i}^{(c)}$ are the weighted sample mean and covariance given by:

$$
\begin{gathered}
W_{0}^{(m)}=\kappa /(n+\kappa) \\
W_{0}^{(c)}=\kappa_{u k f} /\left(n+\kappa_{u k f}\right)+\left(1-\alpha_{u k f}^{2}+\beta_{u k f}\right) \\
W_{i}^{(m)}=W_{i}^{(c)}=\kappa_{u k f} /\left[2\left(n+\kappa_{u k f}\right)\right], \quad i=1,2, \ldots, 2 n
\end{gathered}
$$

and $\beta_{u k f}$ is used to incorporate prior knowledge of the distribution with $\beta_{u k f}=2$ (Crassidis and Junkins 2004). The predicted mean of the state vector $\tilde{\mathbf{x}}_{k}^{-}$, the covariance matrix $\mathbf{P}_{x, k}^{-}$and the mean observation $\tilde{\mathbf{y}}_{k}^{-}$can be approximated using the weighted mean and covariance of the transformed vectors:

$$
\begin{gathered}
\boldsymbol{\mathbf { X }}_{k \mid k-1}^{i}=f\left(\boldsymbol{x}_{k-1}^{i}, \mathbf{u}_{k}\right) \\
\tilde{\mathbf{x}}_{k}^{-}=\sum_{i=0}^{2 n} W_{i}^{(m)} \boldsymbol{\chi}_{k \mid k-1}^{i} \\
\mathbf{P}_{k}^{-}=\sum_{i=0}^{2 n} W_{i}^{(c)}\left[\boldsymbol{\chi}_{k \mid k-1}^{i}-\tilde{\mathbf{x}}_{k}^{-}\right]\left[\boldsymbol{x}_{k \mid k-1}^{i}-\tilde{\mathbf{x}}_{k}^{-}\right]^{T}+\mathbf{Q}_{k} \\
\mathbf{Y}_{k \mid k-1}^{i}=h\left(\boldsymbol{x}_{k \mid k-1}^{i}\right) \\
\tilde{\mathbf{y}}_{k}^{-}=\sum_{i=0}^{2 n} W_{i}^{(m)} \mathbf{Y}_{k \mid k-1}^{i}
\end{gathered}
$$

The updated covariance $\mathbf{P}_{y, k}$ and the cross correlation matrix $\mathbf{P}_{x y, k}$ are:

$$
\begin{aligned}
& \mathbf{P}_{y, k}=\sum_{i=0}^{2 n} W_{i}^{(c)}\left[\mathbf{Y}_{k \mid k-1}^{i}-\tilde{\mathbf{y}}_{k}^{-}\right]\left[\mathbf{Y}_{k \mid k-1}^{i}-\tilde{\mathbf{y}}_{k}^{-}\right]^{T}+\mathbf{R}_{k} \\
& \mathbf{P}_{x y, k}=\sum_{i=0}^{2 n} W_{i}^{(c)}\left[\boldsymbol{X}_{k \mid k-1}^{i}-\tilde{\mathbf{x}}_{k}^{-}\right]\left[\mathbf{Y}_{k \mid k-1}^{i}-\tilde{\mathbf{y}}_{k}^{-}\right]^{T}
\end{aligned}
$$

Finally, the filter state vector $\hat{\mathbf{x}}_{k}$ and covariance updated matrix $\mathbf{P}_{x, k}$ are represented as follows:

$$
\begin{gathered}
\tilde{\mathbf{x}}_{k}=\tilde{\mathbf{x}}_{k}^{-}+\mathbf{K}\left(\mathbf{y}_{k}-\tilde{\mathbf{y}}_{k}^{-}\right) \\
\left(\mathbf{P}_{k}^{+}\right)^{-1}=\left(\mathbf{P}_{k}^{-}\right)^{-1}+\left(\mathbf{P}_{k}^{-}\right)^{-1} \mathbf{P}_{x y, k} \mathbf{R}_{k}^{-1}\left[\left(\mathbf{P}_{k}^{-}\right)^{-1} \mathbf{P}_{x y, k}\right]^{T}-\vartheta_{k} \mathbf{I}_{d} \\
\mathbf{K}=\mathbf{P}_{x y, k} \mathbf{P}_{y, k}^{-1}
\end{gathered}
$$

where $\mathbf{K}$ is the Kalman gain matrix, $\vartheta_{k}$ is the performance bound of the $H_{\infty}$ filter, and $\mathbf{R}_{\mathrm{k}}$ is a suitable matrix which, in the case of a normal distribution, coincides with the measurement noise 
covariance matrix at time step $k$. In order to assure that the covariance matrix is positive definite this value is calculated at each iteration as:

$$
\vartheta_{k}^{-1}=\xi \max \left(\operatorname{eig}\left(\left(\mathbf{P}_{k}^{-}\right)^{-1}+\left(\mathbf{P}_{k}^{-}\right)^{-1} \mathbf{P}_{x y, k} \mathbf{R}_{k}^{-1}\left[\left(\mathbf{P}_{k}^{-}\right)^{-1} \mathbf{P}_{x y, k}\right]^{T}\right)^{-1}\right)
$$

with $\xi$ a scaling parameter. For small values of $\vartheta_{k}$ the terms $\vartheta_{k} \mathbf{I}_{d}$ tends to 0 , and the covariance update in Eq. (54) is equivalent to the one in the UKF. As one can see from the set of equations (53) to (55), the performance bound has no direct effect on the calculation of the gain and on the update step for the estimated state. Nonetheless $\vartheta_{k}$ modifies the shape of covariance matrix update in Eq. (54), which, in turn, generates a different distribution of the sigma points. In this way, the propagation and the update step at the following time step will be directly influenced by the value of the performance bound.

Figure 6 shows an example of the combined filter and controller process for Spacecraft 3 with different levels of initial position error (expressed as a percentage of the nominal initial distance from the asteroid). Being the closest spacecraft to the asteroid, Spacecraft 3 experiences the highest level of perturbations from the gravity field of the asteroid. The controller gain $\kappa$ is set again as $10^{-6} / \mathrm{s}^{2}$ while the steady dissipative coefficient $c_{d}$ is $10^{-3} / \mathrm{s}$. The position error for initial filter guess was set equal to $10 \%$ of the actual position vector while the velocity error was $1 \mathrm{~mm} / \mathrm{s}$ on each component of the velocity. The whole process was simulated for 1 day. The measurements were taken every $600 \mathrm{~s}$.

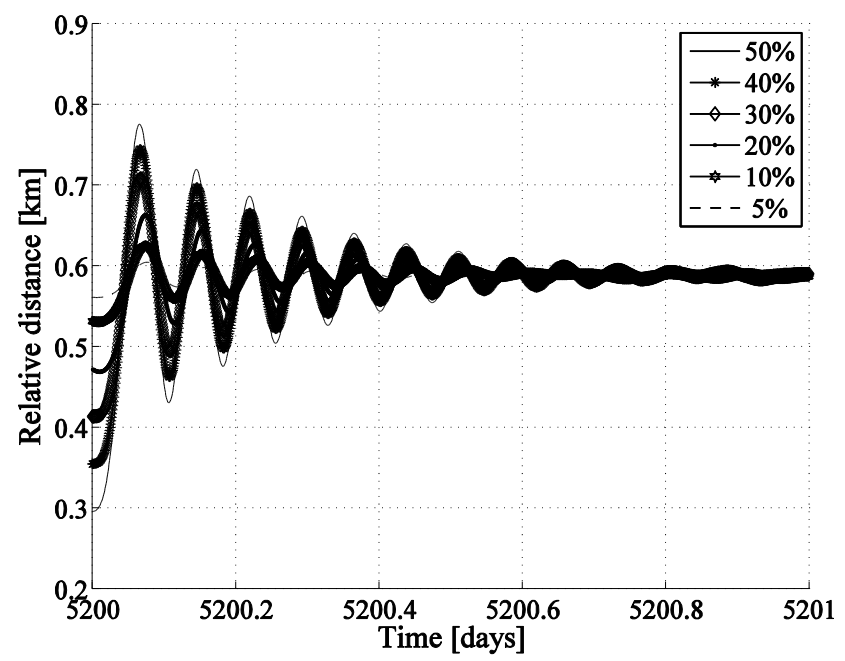

Figure 6. Relative distance for different level of initial position error for Spacecraft 3.

In this case the spacecraft could rely on the full set of measurements including intersatellite measurements. The error on range $s_{1}$ is drawn from a normal distribution with zero mean and 10 $\mathrm{m}$ of standard deviation. The pixelization error $\varsigma_{p}$ was set to $75 \mu \mathrm{m}$, the size of a pixel, for each of the points selected in the centroiding process. The attitude error $\lambda_{1,2}$ in Eq. (15) was drawn from a normal distribution with zero mean and $10^{-3}$ rad of standard deviation. The intersatellite errors $d_{r e l}$ and $\varsigma_{\theta_{\text {rel }}, \varphi_{\text {rel }}}$ were drawn from two normal distributions with zero mean and standard deviations $2 \mathrm{~m}$ and $10^{-3}$ rad respectively. We defined $\mathbf{R}_{\mathrm{k}}$ as a constant diagonal matrix with components equal to the inverse of the measurement covariance and used the same value for both the Kalman and $H_{\infty}$ filters as in Li and Jia (2010). The only exception to this definition was for the 
elements of $\mathbf{R}_{\mathrm{k}}$ corresponding to the error in the camera pointing angles, $\varsigma_{\psi, \varphi}$ in Eq. (23), which were equal to $2.6 \times 10^{-3} \mathrm{rad}$. As one can see, after an initial transient response, the spacecraft is able to gain the nominal distance from the asteroid in less than 1 day even when the initial position error is up to $300 \mathrm{~m}$ from the nominal initial position.

In the remainder of this analysis it will be shown that the UHF has superior performance compared to the classical UKF, EKF and EHF. As an example, consider the case of Spacecraft 1 flying in the proximity of the asteroid for 2 days. The process is simulated 100 times to collect a statistically meaningful sample to assess the performance of the filters. Differently from the previous example, in this case, the spacecraft cannot rely on the intersatellite measurements. Furthermore, a bias is introduced in all measurements: the error on range $\varsigma_{1}$ is biased by $10 \mathrm{~m}$ and has a standard deviation of $50 \mathrm{~m}$. The identification of the pixel on the camera was the same as in the previous case. The attitude error $\lambda_{1,2}$ was again drawn from a normal distribution with zero mean and $10^{-3} \mathrm{rad}$ of standard deviation, this time biased by $10^{-3} \mathrm{rad}$. The position error for initialising the filter was taken as $20 \%$ of the nominal position vector component, while the velocity error was $1 \mathrm{~cm} / \mathrm{s}$ on each components of the velocity. The scaling parameter for UHF was $\xi=35$. The initial spacecraft displacement was set to $10 \%$ of the nominal initial position vector. Matrix $\mathbf{R}_{\mathrm{k}}$ was defined as a constant diagonal matrix with components equal to the ones of the previous case.

Figure 7 shows the results of the orbit determination for the EHF and UKF while Figure 8 shows the ones for EHF and UHF. Both results are expressed in terms of root mean square error (RMSE) plus 1-sigma dispersion with respect to the mean value.

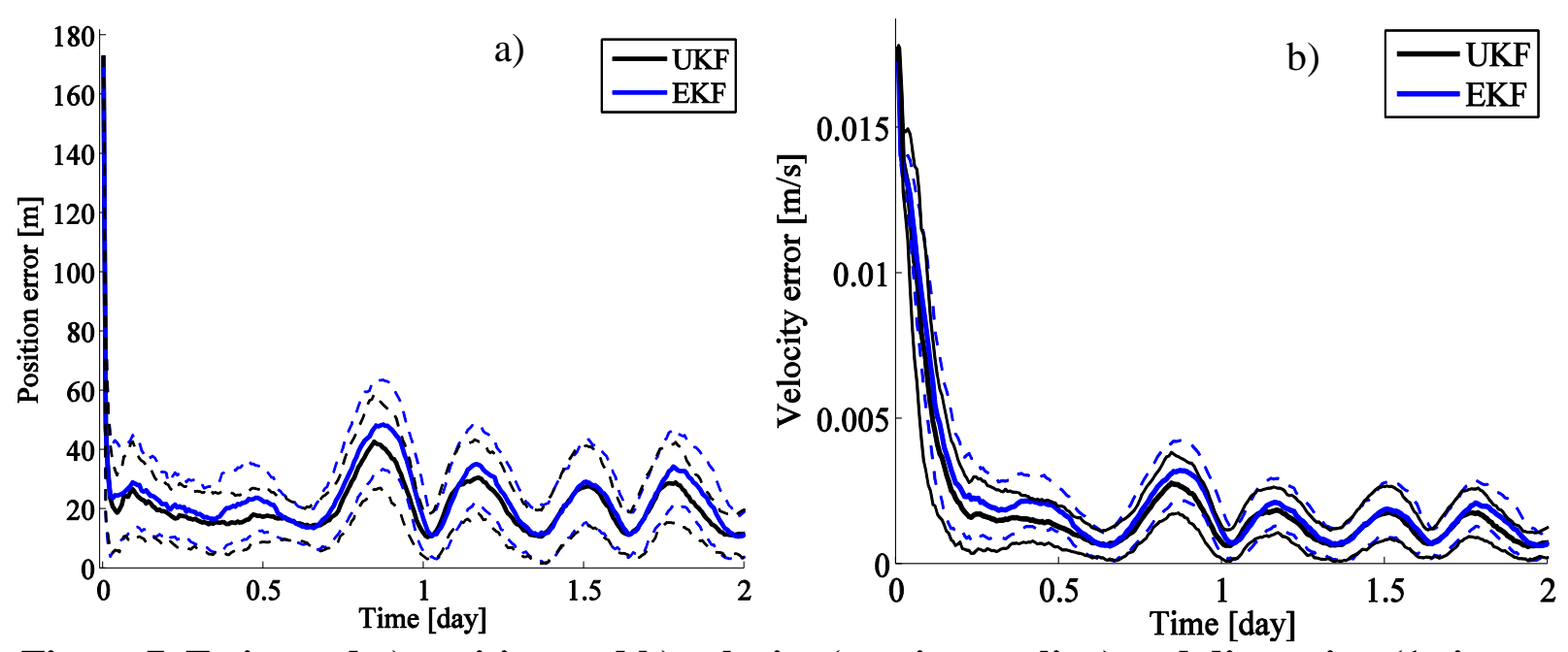

Figure 7. Estimated a) position and b) velocity (continuous line) and dispersion (1-sigma, dashed line) for UKF and EKF. 

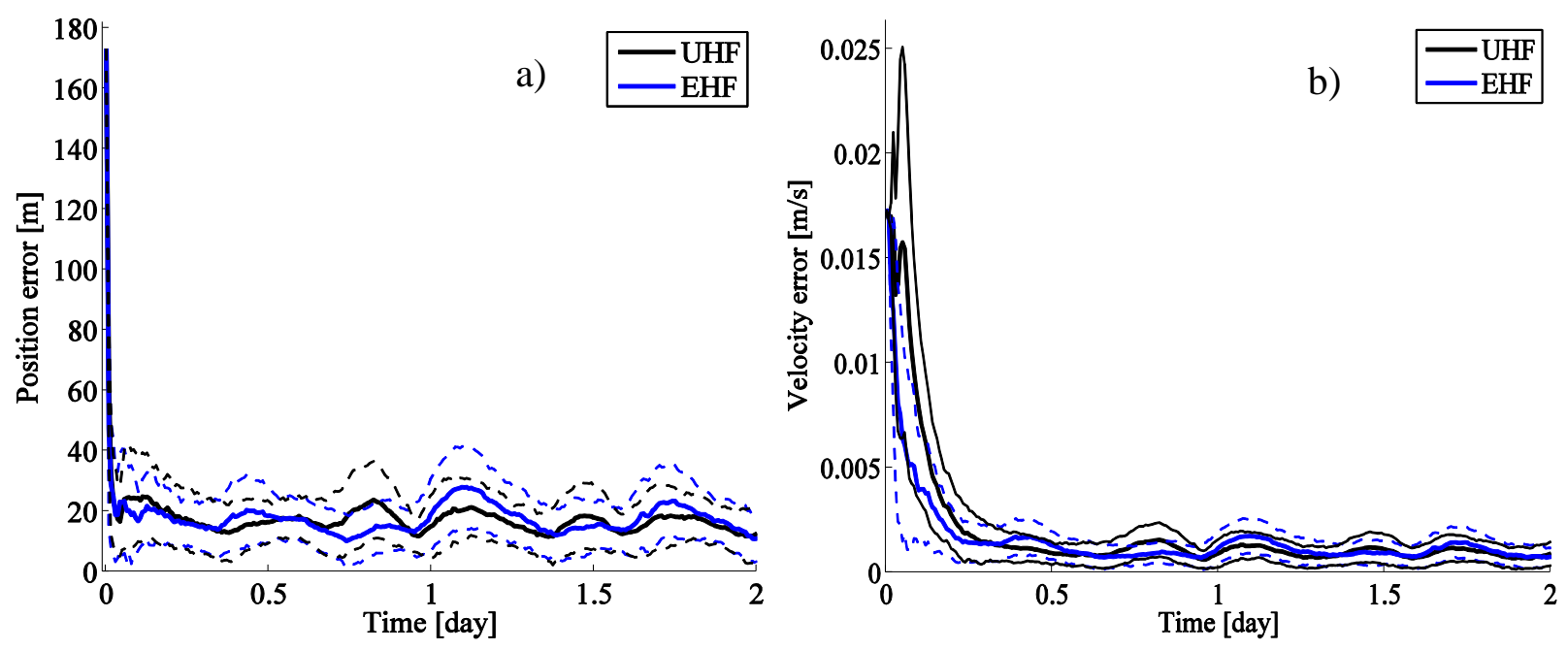

Figure 8. Estimated a) position and b) velocity RMSE (continuous line) and dispersion (1sigma, dashed line) for UHF and EHF.

From the figures one can see that the estimated position and velocity errors differ significantly between the UKF and UHF and between the EKF and the EHF. The UHF appears better, although marginally, than the EHF, in particular in the controlled case. The reason can be found in the coupled interaction between state estimation and control process. In fact a small variation in the estimated state causes the controller to apply a higher or lower thrust level. This causes the non-linear dynamics to produce quite diverse trajectories, see Figure 9.
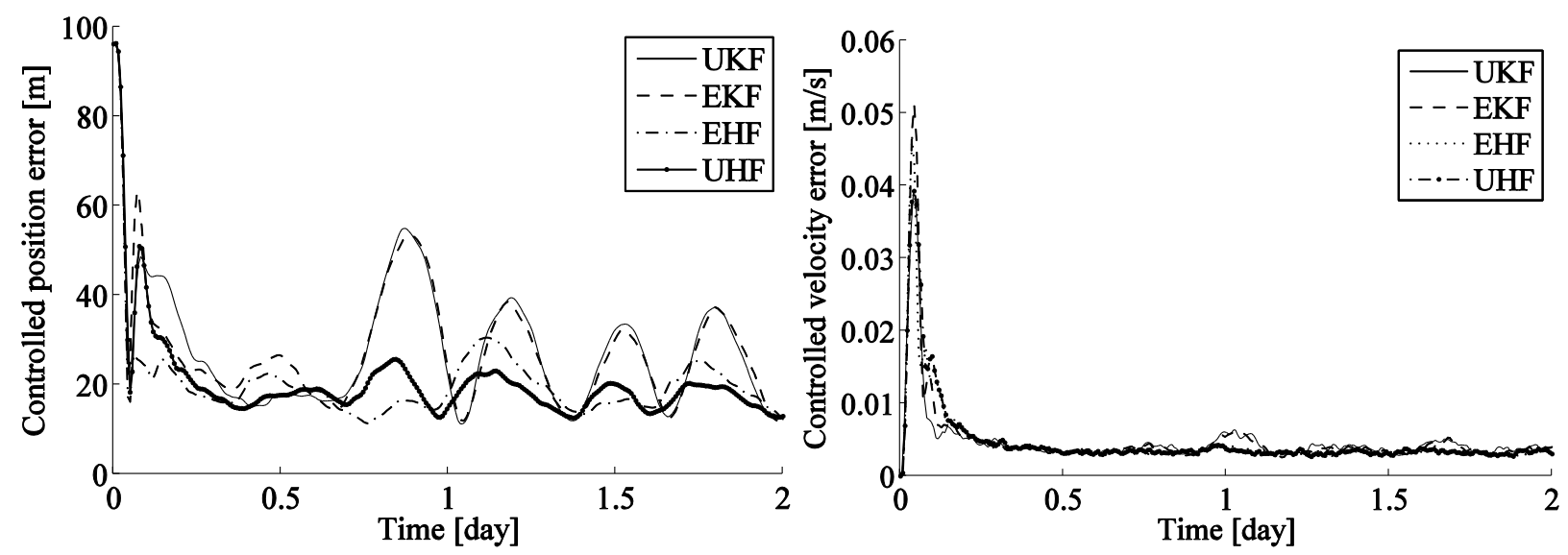

Figure 9. Controlled a) position and b) velocity average RMSE for UKF, EKF, UHF and EHF.

We based the selection of the filter technique on the average RMSE and on the maximum steady state expected error (1-sigma) after 1 day of operation, reported in Table 3. As one can see, the UHF presents superior performance compared to the other filters both in terms of estimated and controlled trajectory. The UHF is more accurate than the UKF with a RMSE that is $25 \%$ lower in position and about $30 \%$ in velocity. When one considers the maximum expected errors, the UHF also presents the best results. A further confirmation comes from the Wilcoxon signed-rank test (Gibbons and Chakraborti 2011) applied to the results of the different filters. When two samples overlap completely the Wilcoxon signed-rank test reports 1 , while 0 is obtained when the 
samples are completely independent. When we cross-checked the samples from the Kalman against the $\mathrm{H}$-infinity filters, the results was below $10^{-18}$, while the cross-check between the UKF and EKF gave 0.643 and the one between UHF and EHF was 0.538. This confirms that the realizations produce different results for the Kalman and $\mathrm{H}$-infinity families on this particular problem.

One advantage of the UHF over the EHF is that it does not require the derivation and propagation of the Jacobian matrix, similarly to the UKF with the EKF (Crassidis and Junkins 2004). Although the computational cost is $20 \%$ greater than for the EHF, nonetheless we considered the UHF as baseline filter because of its higher accuracy in the estimation of the fully controlled trajectory.

Table 3. Average RMSE comparison for different filters - SC1

\begin{tabular}{|c|c|c|c|c|}
\hline & \multicolumn{4}{|c|}{ Filter technique } \\
\hline & UKF & EKF & EHF & UHF \\
\hline CPU time [s] & 1086 & 834 & 906 & 1140 \\
\hline $\begin{array}{c}\text { RMSE position } \\
(\text { max 1- } \sigma)[\mathrm{m}]\end{array}$ & $24.03(43.53)$ & $24.30(48.56)$ & $18.18(41.88)$ & $17.78(31.82)$ \\
\hline $\begin{array}{c}\text { RMSE velocity } \\
(\text { max 1- } \sigma)[\mathrm{mm} / \mathrm{s}]\end{array}$ & $2.27(2.71)$ & $2.41(2.97)$ & $1.88(2.60)$ & $1.59(2.03)$ \\
\hline $\begin{array}{c}\text { RMSE controlled position } \\
(\text { max 1- } \sigma)[\mathrm{m}]\end{array}$ & $27.27(47.56)$ & $27.89(52.82)$ & $20.50(45.23)$ & $20.39(34.36)$ \\
\hline $\begin{array}{c}\text { RMSE controlled velocity } \\
(\text { max 1- } \sigma)[\mathrm{mm} / \mathrm{s}]\end{array}$ & $4.67(9.34)$ & $4.90(9.64)$ & $4.67(7.51)$ & $4.63(6.5)$ \\
\hline
\end{tabular}

\subsection{Multi-spacecraft Data Fusion Process}

Having defined the filtering and control processes, each spacecraft needs to data fuse its own measurements and the information shared with the other spacecraft. This section describes the data fusion process implemented to address this issue.

Each spacecraft receives the whole set of measurements coming from all the members and builds the necessary matrices for the filtering process. It is assumed that the measurements are all received at the same time. If not the measurements may be unavailable at a certain stage of the simulation. This would affect the forecasting and the updating stages, since it would introduce inconsistencies between the forecasted measurements and the measurements that the system actually receives.

The data fusion management can be described through the following 5 main steps:

1. At initial time $t_{0}$, an initial state vector and covariance matrix are assembled from the initial guess $\tilde{\mathbf{x}}_{0}^{i}$ and covariance $\mathbf{P}_{0}^{i}$ of each spacecraft $\left(i=1: N_{s c}\right)$ :

$$
\begin{aligned}
& \mathbf{x}_{0}=\left[\begin{array}{lll}
\tilde{\mathbf{x}}_{0}^{1} & \cdots & \tilde{\mathbf{x}}_{0}^{N_{s c}}
\end{array}\right] \\
& \mathbf{P}_{0}=\left[\begin{array}{lll}
\mathbf{P}_{0}^{1} & & \varnothing \\
& \ddots & \\
\varnothing & & \mathbf{P}_{0}^{N_{s c}}
\end{array}\right]
\end{aligned}
$$

At each time $t_{k}$ (for $k=1,2 \ldots$ ) a set of measurements is received, a total array of measurements is then assembled along with error covariance matrix using the available 
measurements $\mathbf{z}_{k}^{i}$ and instruments covariance error $\mathbf{R}_{0}^{i}$ (with the only exception of the camera angles where we used $2.6 \times 10^{-3} \mathrm{rad}$ ):

$$
\begin{aligned}
& \mathbf{z}_{k}=\left[\begin{array}{lll}
\mathbf{z}_{k}^{1} & \cdots & \mathbf{z}_{k}^{N_{s c}}
\end{array}\right] \\
& \mathbf{R}_{k}=\left[\begin{array}{lll}
\mathbf{R}_{k}^{1} & & \varnothing \\
& \ddots & \\
\varnothing & & \mathbf{R}_{k}^{N_{s c}}
\end{array}\right]
\end{aligned}
$$

2. Based on the type of measurement the unperturbed set of equations $\mathbf{h}_{\mathbf{k}}(\mathbf{x}, t)$ is defined on the basis of the model introduced in Section 3.

3. The UHF is then employed between the two instants $\left(t_{\mathrm{k},} t_{\mathrm{k}+1}\right)$, obtaining the filter gain and the predicted state vector and measurements at time $t_{\mathrm{k}+1}$.

4. At time $t_{\mathrm{k}+1}$, predicted and actual measurements are available. If the number of measurements is lower than the predicted number, only the consistent measurements between the two steps are considered in the update step. This is obtained either by removing the predicted measurements and the correspondent columns and rows in the filter gain or by giving a null value to the correspondent elements in the filter gain. If the number of actual measurements at time $k+1$ is higher than the one at the previous instant, then $\mathbf{R}_{k}$ and $\mathbf{h}_{\mathbf{k}}(\mathbf{x}, t)$ are consistently redefined and steps 2 to 4 are repeated.

\section{Results}

The simulations in this section assume that a formation of 4 spacecraft is flying in the proximity of Apophis. The effects of perturbations would cause the spacecraft to leave the formation with the asteroid if no control was provided.

The camera is assumed to have a resolution of $2048 \times 2048$ pixels, with a field of view of 30 degrees and the focal length of $300 \mathrm{~mm}$. Table 3 summarizes the measurement errors used in the simulations. The LIDAR range error is set to $10 \mathrm{~m}$ according to Kubota et al. (2003), and a precision of $2 \mathrm{~m}$ is used for the inter-spacecraft LIDAR range measurement error. Angular measurements and attitude errors are from Yim et al. (2000).

Table 4. Sensors error for close proximity navigation.

\begin{tabular}{cccc}
\hline & Parameter variation (1-sigma) & \\
\hline & Precision & Worst Case Precision & Bias \\
\hline Camera Pixel $\varsigma_{p}[\mu \mathrm{m}]$ & 75 & $/$ & $/$ \\
LIDAR $\varsigma_{1}[\mathrm{~m}]$ & 10 & 50 & 1 \\
Intersatellite Distance $\varsigma_{d}[\mathrm{~m}]$ & 2 & 10 & 1 \\
Intersatellite Angles $\varsigma_{r_{\text {rel }}, \varphi_{\text {rel }}}[\mathrm{rad}]$ & $10^{-3}$ & $/$ & $5 \cdot 10^{-4}$ \\
Attitude $\lambda_{1,2}[\mathrm{rad}]$ & $10^{-3}$ & $/$ & $5 \cdot 10^{-4}$ \\
\hline
\end{tabular}

(C: camera, I: intersatellite)

For the analysis of the improvement of the asteroid ephemerides, it is assumed that the measurements from Earth are taken from the ground station of Malindi $\left(-2.9956^{\circ} \mathrm{N}\right.$ latitude and $40.1945^{\circ} \mathrm{E}$ longitude). Typical errors considered in this paper are given in Table 5. Range and 
range rate noise are from Thornton and Border (2003) while a Doppler shift sensor's accuracy and pointing errors are equal to the ones used in Yim et al. (2000).

Table 5. Measurements error for asteroid refinement (1-sigma).

\begin{tabular}{ccc}
\hline Characteristics & Unit & Value \\
\hline Camera pointing angles & $\mathrm{rad}$ & $1.7 \cdot 10^{-5}$ \\
G-Range & $\mathrm{m}$ & 20 \\
G-Range rate & $\mathrm{mm} / \mathrm{s}$ & 0.5 \\
Doppler shift & $\mathrm{mm} / \mathrm{s}$ & 0.1 \\
\hline
\end{tabular}

(G: Ground station)

\subsection{Non-Collaborative vs. Collaborative Case}

First we show that the collaboration among the members of the formation improves the accuracy of the estimates. The simulation spans 2 days with a time interval between measurements of 10 minutes. We repeated the test 100 times to assess the statistical relevance of the results. An initial uncertainty of $\max 20 \%$ in the position vector components and $10^{-5} \mathrm{~km} / \mathrm{s}$ on the velocity vector components, with respect to the reference trajectory, were considered for all the simulations. $\boldsymbol{P}_{0}$ is a diagonal matrix with elements equal to the square of the initial state uncertainty for each spacecraft. The process noise covariance matrix $\mathbf{Q}$ was set to $10^{-24} \times \mathrm{I}_{6 \times 6}$ for the non-collaborative case and $10^{-24} \times \mathrm{I}_{12 \times 12}$ for the collaborative one. The observation error covariance matrix $\mathbf{R}$ is a square and diagonal matrix with the square of observation noise on its diagonal terms. In these two cases we considered that the sensors were working at nominal conditions as reported in Table 4 . The process noise $\mathbf{w}$ and the measurement noise $\mathbf{s}$ were assumed to be uncorrelated with each other and with their previous values over time.

Figure 10 and Figure 11 report the results for the non-collaborative and collaborative case respectively. Figure 10a, b and Figure 11a, b refers to the RMSE for the estimated position and velocity while Figure 10c, d and Figure 11c, d shows the RMSE for the controlled trajectory.
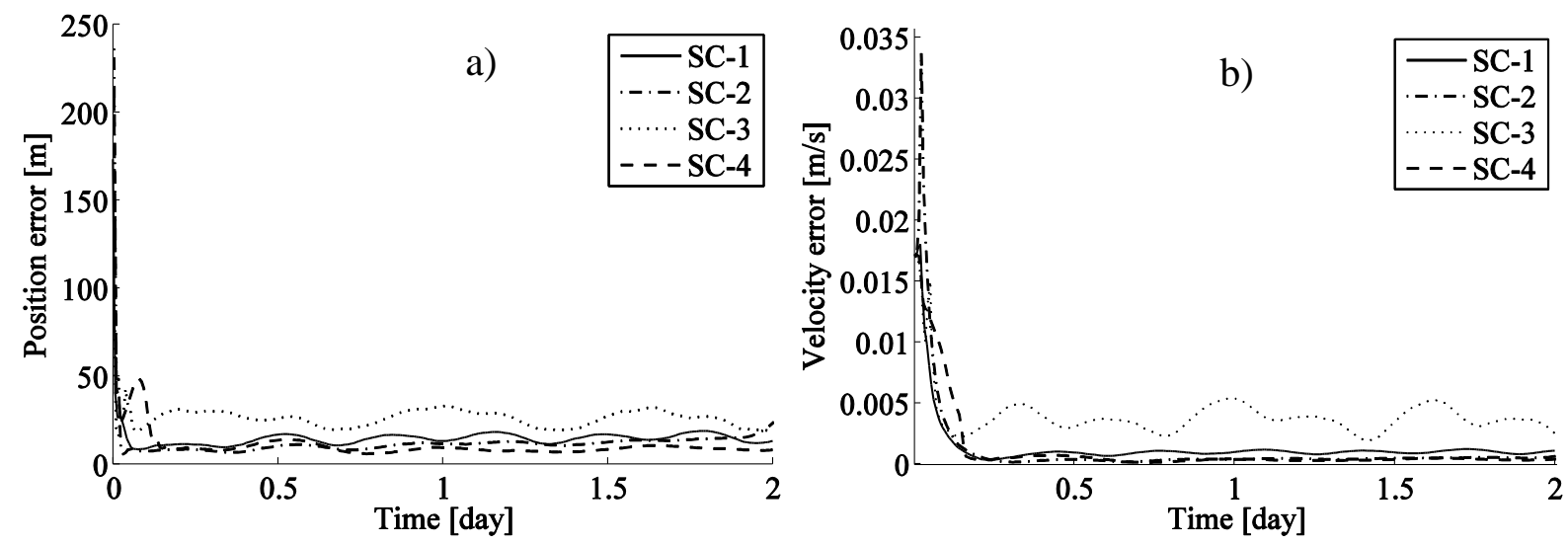

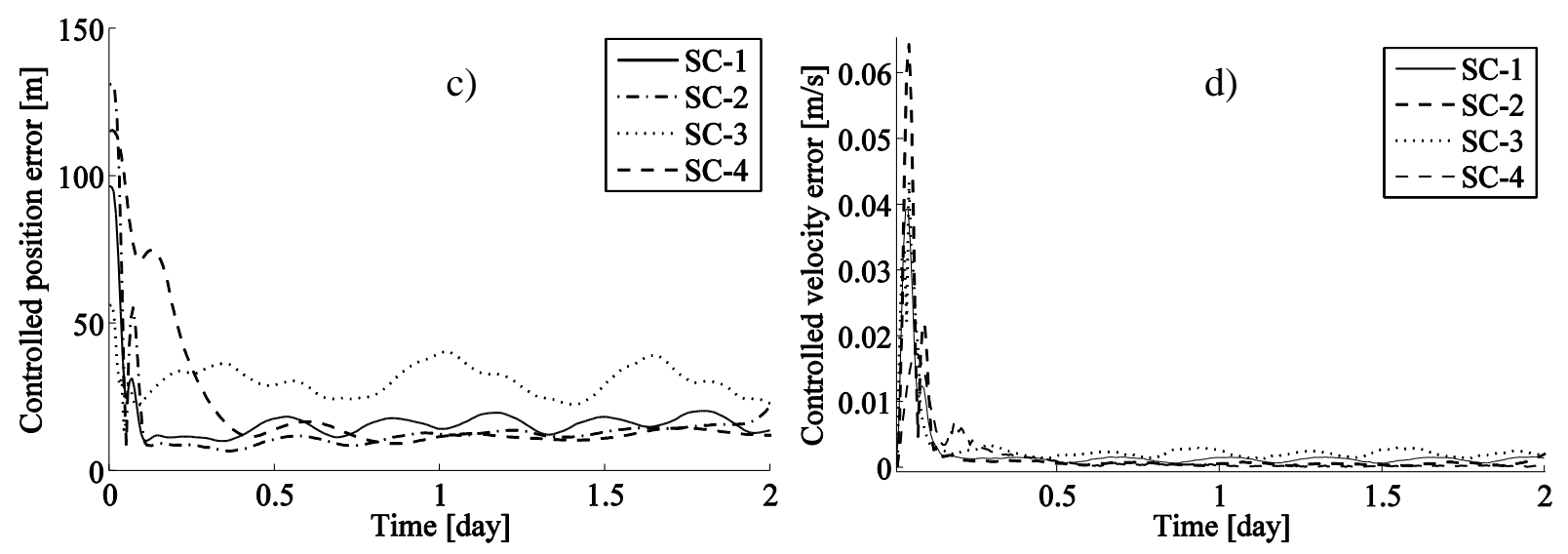

Figure 10. Non-Collaborative case: RMSE for estimated a) position, b) velocity, c) controlled position and d) controlled velocity.
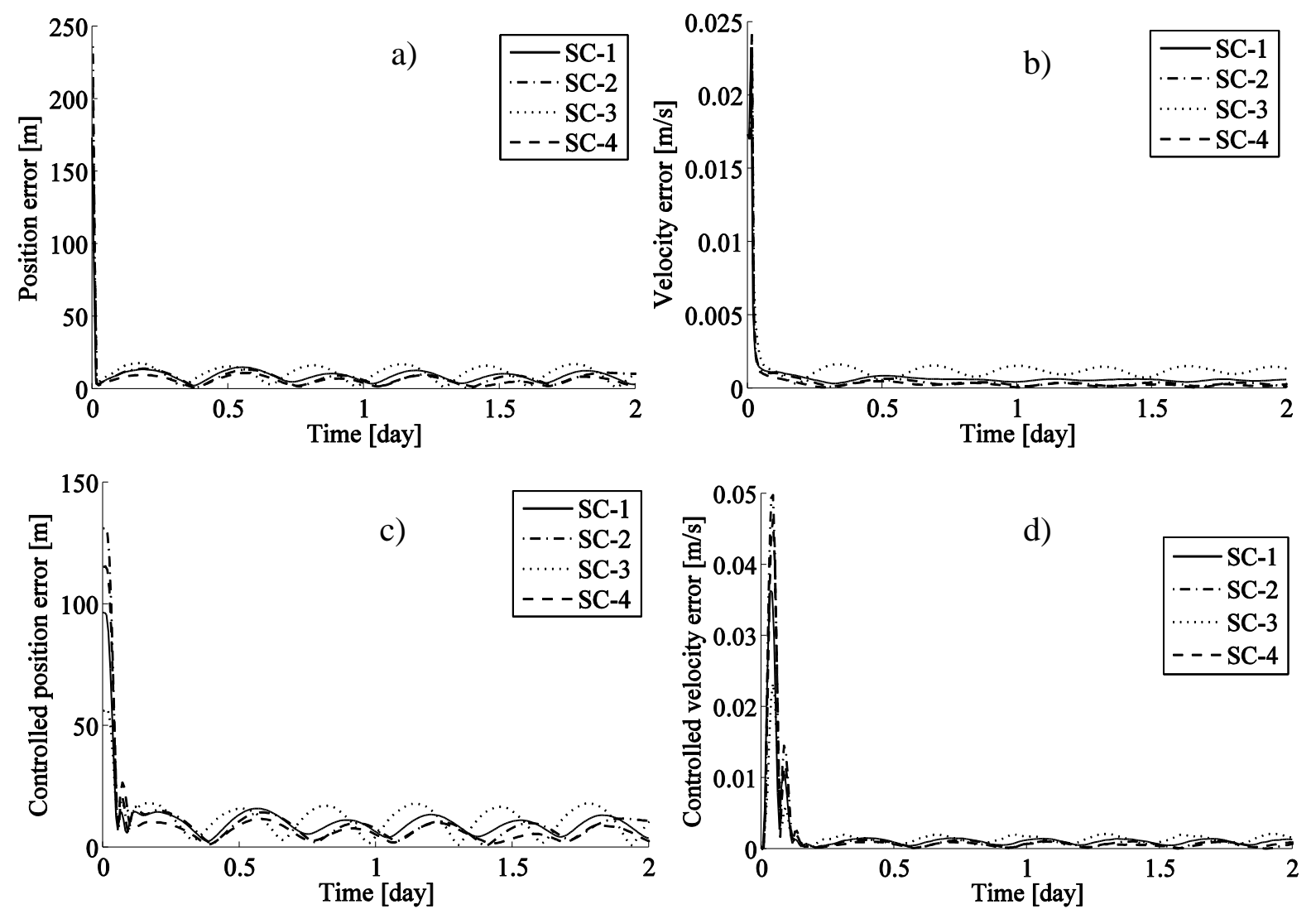

Figure 11. Collaborative case: RMSE for estimated a) position, b) velocity, c) controlled position and d) controlled velocity.

In the non-collaborative case the error is higher than in the collaborative case. In general the initial peaks in the controlled velocity are higher for SC-2 and SC-4 because the control exerts a thrust proportional to the position error. Given the initial conditions for the estimated and the actual trajectory, the control increases the velocity to cope with the actual trajectory deflection. 
SC-3 experiences the worst convergence in terms of accuracy in the estimated and controlled trajectory. When the intersatellite measurements are included, the convergence is improved both in terms of accuracy and time for all the spacecraft. In fact from Figure 11a, d one can see how the error moves towards lower RMSE regions in about 0.1 day with respect to 0.5 day of Figure $10 \mathrm{c}, \mathrm{d}$. The magnitude of the oscillations is reduced, and the initial peaks in the estimated and controlled velocity results are lower than the non-collaborative case (see Figure 10b, $d$ and Figure $11 \mathrm{~b}, \mathrm{~d})$. For the non-collaborative case the maximum error in the estimated velocity is about 3.5 $\mathrm{cm} / \mathrm{s}$ (see Figure 10b) while this value reduces to circa $2.5 \mathrm{~cm} / \mathrm{s}$ with collaboration (Figure $11 \mathrm{~b}$ ). Similarly the collaboration reduces the maximum error in the controlled velocity to about $5 \mathrm{~cm} / \mathrm{s}$ (Figure 10d), compared to the $6.5 \mathrm{~cm} / \mathrm{s}$ as in the non-collaborative case (Figure 11d).

Table 5 reports the average RMSE of each spacecraft. As one can see, the worst performance is achieved by SC-3, which experiences the higher level of perturbations being the closest to the asteroid. When additional information from the intersatellite measurements is added, the improvement is quite considerable especially for SC-3, with the maximum RMSE in the controlled position equal to less than $50 \%$ over the non-collaborative case. For the noncollaborative case the maximum error in the estimated position is $37.70 \mathrm{~m}$, while the collaboration reduces this value to $17.61 \mathrm{~m}$. Similarly the maximum error in the controlled position is reduced to about $18.56 \mathrm{~m}$ from $45.57 \mathrm{~m}$.

Table 5. Average RMSE in estimated and controlled trajectory for the collaborative and un-collaborative case

\begin{tabular}{|c|c|c|c|}
\hline & & No-collaboration & Collaboration \\
\hline \multirow{4}{*}{$\mathrm{SC} 1$} & position/max $1-\sigma[\mathrm{m}]$ & $14.77 / 20.72$ & $9.35 / 12.62$ \\
\hline & velocity/max $1-\sigma[\mathrm{mm} / \mathrm{s}]$ & $1.4 / 1.31$ & $0.8 / 0.62$ \\
\hline & controlled position/max $1-\sigma[\mathrm{m}]$ & $16.94 / 22.32$ & $11.05 / 13.46$ \\
\hline & controlled velocity/max $1-\sigma[\mathrm{mm} / \mathrm{s}]$ & $2.1 / 1.94$ & $1.7 / 1.45$ \\
\hline \multirow{4}{*}{$\mathrm{SC} 2$} & position/max $1-\sigma[\mathrm{m}]$ & $12.65 / 24.93$ & $9.05 / 11.76$ \\
\hline & velocity/max $1-\sigma[\mathrm{mm} / \mathrm{s}]$ & $1.2 / 0.70$ & $0.6 / 0.45$ \\
\hline & controlled position/max $1-\sigma[\mathrm{m}]$ & $14.99 / 22.67$ & $11.06 / 12.29$ \\
\hline & controlled velocity/max $1-\sigma[\mathrm{mm} / \mathrm{s}]$ & $2.2 / 2.52$ & $1.9 / 1.29$ \\
\hline \multirow{4}{*}{ SC3 } & position/max $1-\sigma[\mathrm{m}]$ & $26.73 / 37.70$ & $11.60 / 17.61$ \\
\hline & velocity/max $1-\sigma[\mathrm{mm} / \mathrm{s}]$ & 4.1/6.1 & $1.4 / 1.59$ \\
\hline & controlled position/max $1-\sigma[\mathrm{m}]$ & $30.81 / 45.57$ & $12.45 / 18.56$ \\
\hline & controlled velocity/max $1-\sigma[\mathrm{mm} / \mathrm{s}]$ & $3.2 / 4.3$ & $1.8 / 2.31$ \\
\hline \multirow{4}{*}{$\mathrm{SC} 4$} & position/max $1-\sigma[\mathrm{m}]$ & $11.56 / 13.58$ & $7.04 / 9.88$ \\
\hline & velocity/max $1-\sigma[\mathrm{mm} / \mathrm{s}]$ & $1.2 / 0.67$ & $0.5 / 0.40$ \\
\hline & controlled position/max $1-\sigma[\mathrm{m}]$ & $20.93 / 18.20$ & $8.85 / 10.43$ \\
\hline & controlled velocity/max $1-\sigma[\mathrm{mm} / \mathrm{s}]$ & $1.5 / 1.7$ & $1.6 / 1.13$ \\
\hline
\end{tabular}

\subsection{Failures}

In order to assess the increased flexibility and robustness provided by intersatellite link, the following contingency scenarios were analysed:

1. LIDAR and camera fail on SC-1;

2. LIDAR and camera fail on SC-1 and SC-2; 
3. LIDAR and camera fail on SC-1 and poor LIDAR on SC-4;

4. LIDAR and camera fail on SC-1 and SC-2 and poor LIDAR on SC-3 and SC-4;

5. LIDAR and camera fail on SC-1 and SC-2 with poor inter-satellite links on SC-1 and SC-2 and poor LIDAR on SC-3 and SC-4;

Table 7 reports the different test cases used to assess the improvement of the estimates even in absence of measurement or in case of failure by means of the decentralized navigation system.

Table 6. Sensors set on spacecraft formation

\begin{tabular}{lllll}
\hline Case & SC-1 & SC-2 & SC-3 & SC-4 \\
\hline 1 & I & C, L/R, I & C, L/R, I & $\mathrm{C}, \mathrm{L} / \mathrm{R}, \mathrm{I}$ \\
2 & $\mathrm{I}$ & $\mathrm{I}$ & $\mathrm{C}, \mathrm{L} / \mathrm{R}, \mathrm{I}$ & $\mathrm{C}, \mathrm{L} / \mathrm{R}, \mathrm{I}$ \\
3 & $\mathrm{I}$ & $\mathrm{C}, \mathrm{L} / \mathrm{R}, \mathrm{I}$ & $\mathrm{C}, \mathrm{L} / \mathrm{R}, \mathrm{I}$ & $\mathrm{C}, \mathrm{L} / \mathrm{R}^{*}, \mathrm{I}$ \\
4 & $\mathrm{I}$ & $\mathrm{I}$ & $\mathrm{C}, \mathrm{L} / \mathrm{R}^{*}, \mathrm{I}$ & $\mathrm{C}, \mathrm{L} / \mathrm{R}^{*}, \mathrm{I}$ \\
5 & $\mathrm{I}^{*}$ & $\mathrm{I}^{*}$ & $\mathrm{C}, \mathrm{L} / \mathrm{R}^{*}, \mathrm{I}$ & $\mathrm{C}, \mathrm{L} / \mathrm{R}^{*}, \mathrm{I}$ \\
\hline
\end{tabular}

C-camera, L/R-LIDAR, I-inter-satellite, ${ }^{*}$ worst case condition

The initial conditions are the same for the collaborative case and the covariance matrix $\mathbf{P}_{0}$, similarly the process noise covariance $\mathbf{Q}$ and $\mathbf{R}$ are built as before. Figure 12 and Figure 13 report only the trend for SC-3 and SC-4 as they present the worst and best cases in terms of RMSE for all the cases. The results for SC- 1 and SC-2 can be found at the end of the paper in Figure 18 and Figure 19.

The trend for cases 1 to 4 is very similar to the collaborative case, demonstrating that the intersatellite link compensates for poor or incomplete direct measurements. In case 5, instead, spacecraft SC-3 experiences higher levels of error with oscillations due to both the filter and control coupling and perturbations, see Figure 12a and $b$. The controller is not able to converge as well as in the other case, shown in Figure 12c and d.

Figure 13a and $\mathrm{b}$ show that for SC-4, the oscillatory behaviour due to the coupling between filter and controller is less pronounced in cases 1 to 4 . While in case 5, SC-4 has a behaviour comparable to the one of SC-3, with the maximum controlled position and velocity error respectively in the range $30-50 \mathrm{~m}$ and $3-6 \mathrm{~mm} / \mathrm{s}$ (after the initial transient response), see Figure $13 \mathrm{c}$ and $\mathrm{d}$.
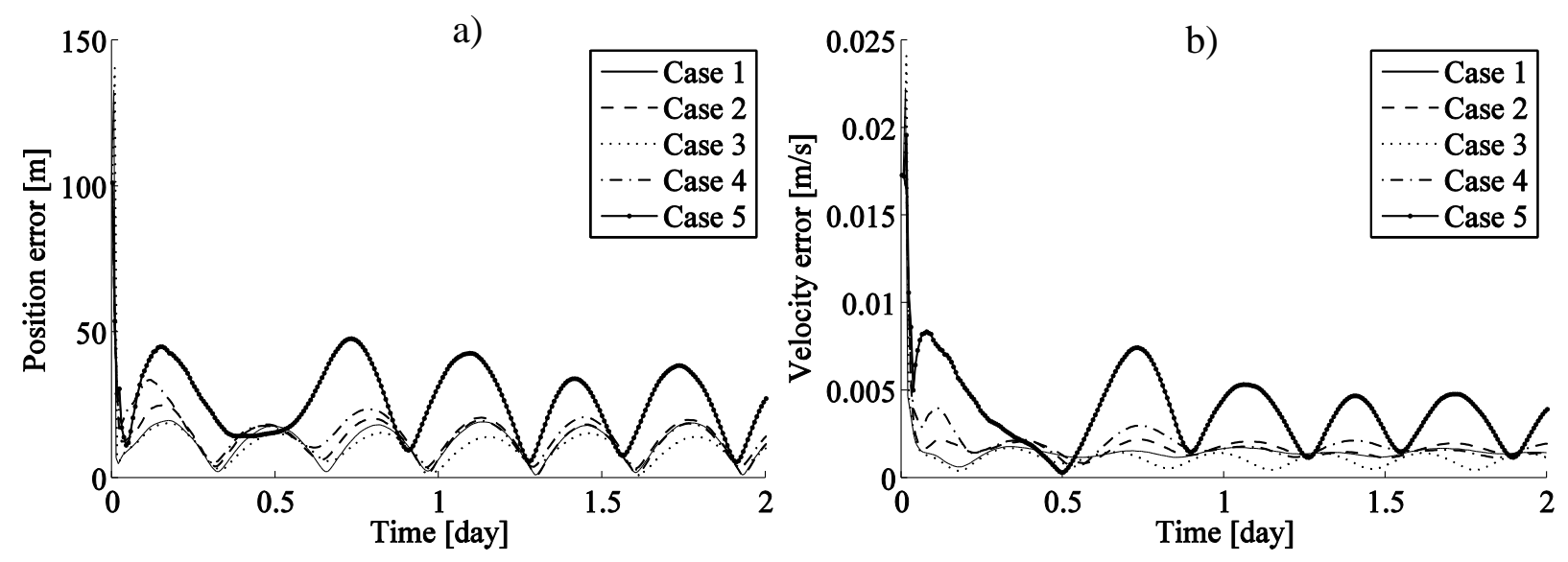

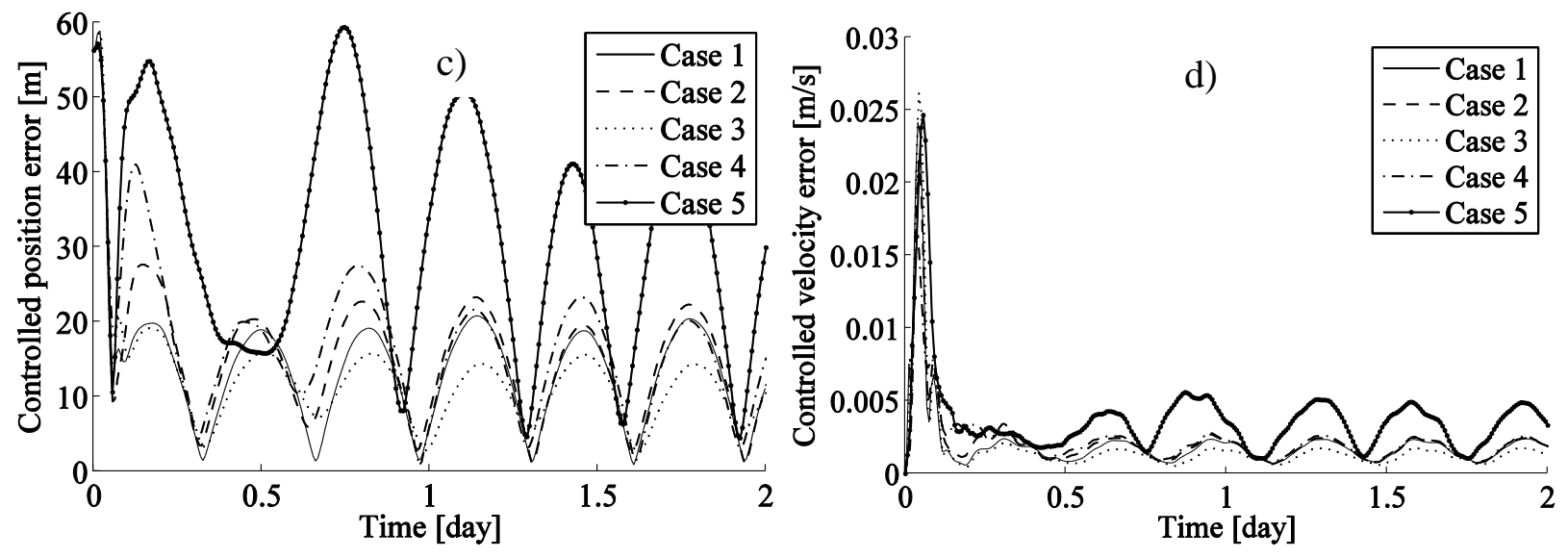

Figure 12. SC-3 failures: RMSE for estimated a) position, b) velocity, c) controlled position and d) controlled velocity.
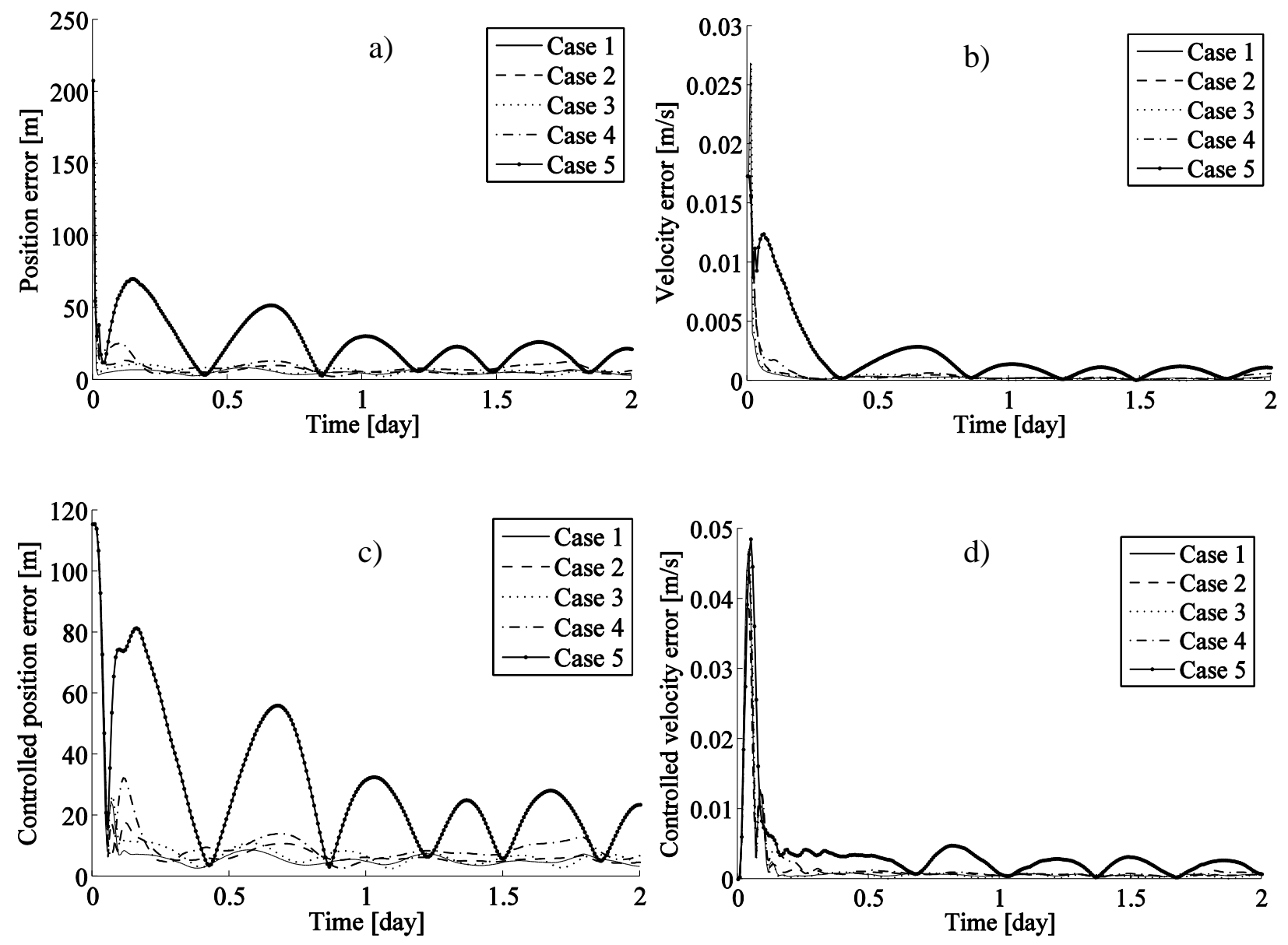

Figure 13. SC-4 failures: RMSE for estimated a) position, b) velocity, c) controlled position and d) controlled velocity. 
The average RMSE of estimated and controlled trajectory of each spacecraft are summarized in Table 7. The results show that the overall performance of the formation is, in general, better than for the non-collaborative case. One thing should be noted: in Cases 1 to 3, some of the spacecraft experience an improvement with respect to the collaborative case with all the instruments working. The explanation of this behaviour resides in the fact that the biases on the camera pointing towards the asteroid introduce a considerable error in the filtering process and the centroid identification is not as precise as the intersatellite measurements. The result is that having less measurements relative to the asteroid has beneficial effects on the mean error.

Cases 4-5 prove that the system is able to determine and maintain the trajectory without losing the proximity with the asteroid but the lack of inter-satellite links has a significant impact on the navigation and control capabilities of the formation.

Table 7. Average RMSE of the estimated and controlled trajectory in case of failures.

\begin{tabular}{|c|c|c|c|c|c|c|}
\hline $\mathrm{SC}$ & & Case 1 & Case 2 & Case 3 & Case 4 & Case 5 \\
\hline \multirow[t]{4}{*}{1} & position/max $1-\sigma[\mathrm{m}]$ & $7.79 / 9.67$ & $9.22 / 11.95$ & $9.50 / 12.84$ & $10.75 / 11.79$ & $17.96 / 19.02$ \\
\hline & $\begin{array}{l}\text { velocity/max } 1-\sigma \\
{[\mathrm{mm} / \mathrm{s}]}\end{array}$ & $0.65 / 0.54$ & $0.70 / 0.68$ & $0.72 / 0.82$ & $0.78 / 0.65$ & $1.51 / 1.05$ \\
\hline & $\begin{array}{l}\text { controlled } \\
\text { position/max } 1-\sigma[\mathrm{m}]\end{array}$ & $9.13 / 10.33$ & $10.51 / 12.86$ & $10.79 / 14.01$ & $12.10 / 12.41$ & $19.99 / 20.63$ \\
\hline & $\begin{array}{l}\text { controlled } \\
\text { velocity/max } 1-\sigma \\
{[\mathrm{mm} / \mathrm{s}]}\end{array}$ & $1.51 / 1.11$ & $1.65 / 1.13$ & $1.79 / 1.30$ & $1.86 / 1.15$ & $2.65 / 2.14$ \\
\hline \multirow[t]{4}{*}{2} & position/max $1-\sigma[\mathrm{m}]$ & $7.97 / 9.24$ & $8.75 / 9.50$ & $9.13 / 11.26$ & $10.58 / 10.13$ & $20.62 / 20.63$ \\
\hline & $\begin{array}{l}\text { velocity/max } 1-\sigma \\
{[\mathrm{mm} / \mathrm{s}]}\end{array}$ & $0.56 / 0.35$ & $0.60 / 0.38$ & $0.62 / 0.48$ & $0.65 / 0.36$ & $1.48 / 2.14$ \\
\hline & $\begin{array}{l}\text { controlled } \\
\text { position/max } 1-\sigma[\mathrm{m}]\end{array}$ & $9.67 / 9.68$ & $10.50 / 10.03$ & $10.89 / 11.94$ & $12.27 / 10.41$ & $23.35 / 24.14$ \\
\hline & $\begin{array}{l}\text { controlled } \\
\text { velocity/max } 1-\sigma \\
{[\mathrm{mm} / \mathrm{s}]}\end{array}$ & $1.72 / 1.07$ & $1.87 / 0.99$ & $1.79 / 1.19$ & $2.00 / 0.99$ & $3.11 / 2.99$ \\
\hline \multirow[t]{4}{*}{3} & position/max $1-\sigma[\mathrm{m}]$ & $12.57 / 19.39$ & $14.11 / 21.37$ & $11.40 / 15.66$ & $15.49 / 21.51$ & $26.89 / 43.97$ \\
\hline & $\begin{array}{l}\text { velocity/max } 1-\sigma \\
{[\mathrm{mm} / \mathrm{s}]}\end{array}$ & $1.61 / 1.77$ & $1.87 / 2.20$ & $1.32 / 1.48$ & $2.08 / 2.26$ & $3.9 / 5.52$ \\
\hline & $\begin{array}{l}\text { controlled } \\
\text { position/max } 1-\sigma[\mathrm{m}]\end{array}$ & $13.63 / 21.19$ & $15.60 / 23.93$ & $12.20 / 15.79$ & $17.13 / 23.95$ & $31.78 / 51.93$ \\
\hline & $\begin{array}{l}\text { controlled } \\
\text { velocity/max } 1-\sigma \\
{[\mathrm{mm} / \mathrm{s}]}\end{array}$ & $1.99 / 2.53$ & $2.17 / 2.71$ & $1.75 / 1.81$ & $2.28 / 2.82$ & $3.79 / 5.56$ \\
\hline \multirow[t]{4}{*}{4} & position/max $1-\sigma[\mathrm{m}]$ & $6.16 / 7.12$ & $7.54 / 6.97$ & $7.64 / 752$ & $10.10 / 13.54$ & $26.39 / 31.60$ \\
\hline & $\begin{array}{l}\text { velocity/max } 1-\sigma \\
{[\mathrm{mm} / \mathrm{s}]}\end{array}$ & $0.47 / 0.31$ & $0.56 / 0.35$ & $0.53 / 0.36$ & $0.65 / 0.66$ & $2.09 / 1.48$ \\
\hline & $\begin{array}{l}\text { controlled } \\
\text { position/max } 1-\sigma[\mathrm{m}]\end{array}$ & $7.75 / 7.39$ & $9.11 / 7.21$ & $9.23 / 8.04$ & $11.43 / 13.59$ & $30.04 / 33.72$ \\
\hline & $\begin{array}{l}\text { controlled } \\
\text { velocity/max } 1-\sigma \\
{[\mathrm{mm} / \mathrm{s}]}\end{array}$ & $1.49 / 0.79$ & $1.66 / 0.82$ & $1.55 / 0.96$ & $1.77 / 1.34$ & $3.59 / 3.49$ \\
\hline
\end{tabular}


Even though the number of failures would not allow Spacecraft 1-2 alone to estimate their trajectories, the collaboration increases the reliability of the system and the robustness against sensors failures.

\subsection{Asteroid Ephemerides Refinement}

During the approach phase, the on-board measurements can be employed in combination with the absolute measurements from the ground station to refine the trajectory of the asteroid. Two different sets of measurements were considered for this analysis: the first set combines ground tracking with line of sight measurements taken from the spacecraft, while the second includes also a Sun Doppler shift sensor among the on-board measurements.

In the following, it is assumed that two spacecraft will approach the asteroid at the same time when the asteroid is at perihelion. In such a situation, if the spacecraft formation travels from the Sun direction, given its visual magnitude of 19.7, the asteroid could be detected from a distance of about 2,000,000 km (Vetrisano et al. 2013). We conservatively assumed that both spacecraft will concurrently start the acquisition of the target at 1,000,000 km from the asteroid.

The configuration of the approach and acquisition phase is depicted in Figure 14. The initial conditions are given with respect to the asteroid's Hill reference frame in terms of distance $d_{a p}$, azimuth $\tau$ and elevation $\lambda$. Both spacecraft are placed at 1,000,000 $\mathrm{km}$ with an approach velocity $v_{a p}$ of $100 \mathrm{~m} / \mathrm{s}$ in magnitude directed along the spacecraft-to-asteroid vector. In the Hill reference frame the position and velocity vectors for the $i^{\text {th }}$ spacecraft can be written as:

$$
\begin{aligned}
& \delta \mathbf{r}_{s c-i}^{h}=\mathbf{r}_{A}^{h}+d_{a p}\left[\begin{array}{llll}
\cos \tau_{i} \cos \lambda_{i} & \cos \tau_{i} \cos \lambda_{i} & \sin \lambda_{i}
\end{array}\right] \\
& \delta \dot{\mathbf{r}}_{s c-i}^{h}=\mathbf{v}_{A}^{h}-v_{a p}\left[\begin{array}{llll}
\cos \tau_{i} \cos \lambda_{i} & \cos \tau_{i} \cos \lambda_{i} & \sin \lambda_{i}
\end{array}\right]
\end{aligned}
$$

where the superscript $h$ refers to the Hill reference frame.

From Figure 14 one can see that if the two spacecraft are separated by a small angle, the asteroid trajectory becomes poorly observable as it is not possible to accurately triangulate the position of the asteroid. Direct distance measurement from the asteroid to the spacecraft cannot be acquired using the LIDAR, neither can the distance be derived from a single camera unless complicated dogleg manoeuvres are adopted, because the shape of the asteroid might not be known in advance or the camera might not have a sufficiently high resolution. Therefore, the angular separation of the two spacecraft, seen from the asteroid, needs to be sufficiently high. 


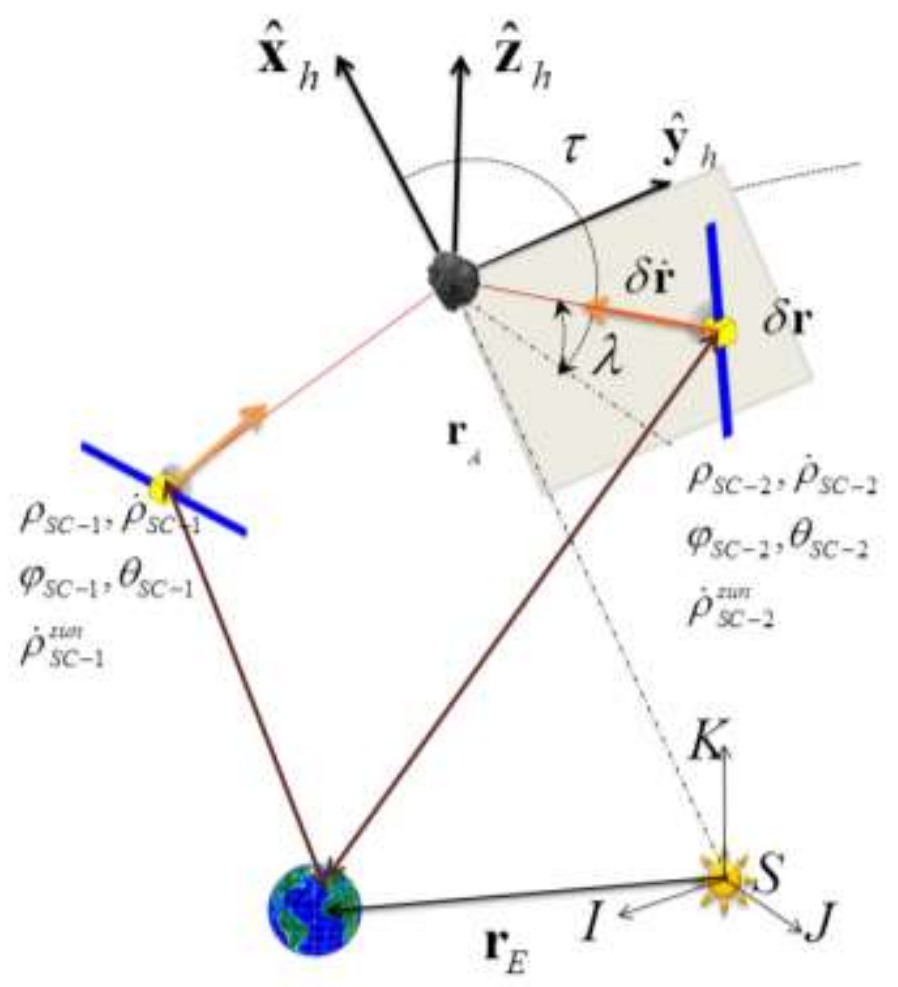

Figure 14. Approach and acquisition phase configuration.

As an example, Figure 15 reports the estimated error with respect to the actual trajectory of the asteroid when only a single spacecraft is used. The measurements are acquired over two weeks. The initial estimated position of the asteroid is randomly taken on a sphere with radius $10000 \mathrm{~km}$ centred on the actual position, while for the estimated velocity a sphere of $2.5 \mathrm{~m} / \mathrm{s}$ radius with respect to the actual velocity is considered. The 1-sigma dispersion in position is $50000 \mathrm{~km}$ and $90 \mathrm{~m} / \mathrm{s}$ in velocity. These error values are higher than the one used in Vetrisano et al. (2013) for a single spacecraft performing a dogleg approach to an asteroid whose trajectory is not precisely known. It is clear that in this case the problem is not observable since the type of information is not sufficient to improve the estimate without a dogleg approach. In fact the error increases with time.

Consider two spacecraft where their positions are known with an initial accuracy of $1000 \mathrm{~km}$ and velocity with an accuracy of $0.1 \mathrm{~m} / \mathrm{s}$ in magnitude. The sensors on-board the spacecraft and the ground station tracking system provide the navigation algorithm with 5 measurements for each spacecraft: line-of-sight angles and Doppler shift, range and range rate from Earth. In total there are up to 10 measurements acquired every 1 hour. 

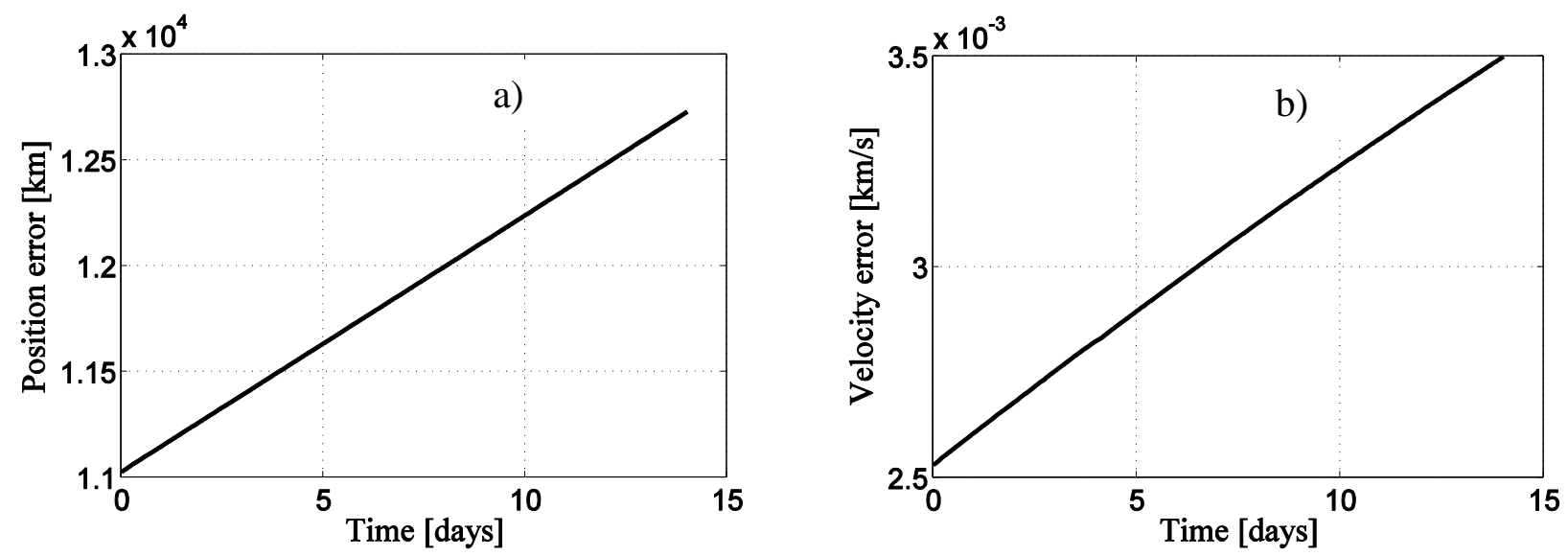

Figure 15. Asteroid estimate a) position and b) velocity error in 14 days using only onboard optical observation from a single spacecraft with ground station tracking.

Table 8 reports the results for different configurations of $\tau$ and $\lambda$, each one simulated 100 times. It is assumed that the measurement acquisition and state estimation processes run for 7 days. The first spacecraft was placed on the nominal orbit plane of the asteroid while the second spacecraft was given a maximum out of plane component equal to the asteroid's initial dispersion in position that corresponds to a value of 3 degrees for $\lambda$.

Table 8. Analysed configurations and final estimated error without and with Doppler shift.

\begin{tabular}{|c|c|c|c|c|c|c|c|c|}
\hline & \multicolumn{7}{|c|}{ Configuration } \\
\hline & & 1 & 2 & 3 & 4 & 5 & 6 & 7 \\
\hline \multirow[b]{2}{*}{ SC-1 } & $\tau[\mathrm{deg}]$ & 90 & 180 & 135 & 135 & 135 & 135 & 135 \\
\hline & $\lambda[\mathrm{deg}]$ & 0 & 0 & 0 & 0 & 0 & 0 & 0 \\
\hline \multirow[b]{2}{*}{ SC-2 } & $\tau[\mathrm{deg}]$ & 270 & 270 & 270 & 139 & 136 & 135.5 & 135.5 \\
\hline & $\lambda[\mathrm{deg}]$ & 3 & 3 & 3 & 3 & 3 & 3 & 0.5 \\
\hline \multirow[t]{2}{*}{$\begin{array}{l}\text { No } \\
\text { Doppler }\end{array}$} & $\begin{array}{l}\text { Position } \\
\text { error }[\mathrm{km}]\end{array}$ & 31.38 & 5.657 & 8.04 & 17.50 & 25.14 & 26.25 & 115.25 \\
\hline & $\begin{array}{l}\text { Velocity } \\
\text { error } \\
{[\mathrm{km} / \mathrm{s}]}\end{array}$ & $\begin{array}{l}1.009 \cdot \\
10^{-4}\end{array}$ & $\begin{array}{l}1.936 \cdot \\
10^{-5}\end{array}$ & $\begin{array}{l}1.961^{-} \\
10^{-5}\end{array}$ & $\begin{array}{l}6.263 . \\
10^{-5}\end{array}$ & $\begin{array}{l}8.010^{-} \\
10^{-4}\end{array}$ & $\begin{array}{l}8.269 \cdot \\
10^{-5}\end{array}$ & $\begin{array}{l}3.749 \\
10^{-4}\end{array}$ \\
\hline \multirow[t]{2}{*}{ Doppler } & $\begin{array}{l}\text { Position } \\
\text { error }[\mathrm{km}]\end{array}$ & 26.89 & 5.791 & 8.09 & 17.09 & 25.67 & 26.48 & 101.97 \\
\hline & $\begin{array}{l}\text { Velocity } \\
\text { error } \\
{[\mathrm{km} / \mathrm{s}]}\end{array}$ & $\begin{array}{l}9.087 \cdot \\
10^{-5}\end{array}$ & $\begin{array}{l}1.905 \cdot \\
10^{-5}\end{array}$ & $\begin{array}{l}1.915 \cdot \\
10^{-5}\end{array}$ & $\begin{array}{l}6.288 \\
10^{-5}\end{array}$ & $\begin{array}{l}8.227 \cdot \\
10^{-5}\end{array}$ & $\begin{array}{l}8.405 \cdot \\
10^{-5}\end{array}$ & $\begin{array}{l}3.581 \cdot \\
10^{-4}\end{array}$ \\
\hline
\end{tabular}

As expected, without the use of the Doppler measurement, when the two spacecraft are almost aligned along the same spacecraft-to-asteroid vector (see configurations 1-4-5-6-7), the estimated error, both in position and in velocity, is higher than when they are not (see cases 2 and 3). When the Doppler measurement is added, there is a general improvement of these estimates, especially for cases 1 and 7. However, for other configurations, the use of the Sun Doppler shift measurements does not lead to a noticeable improvement in the results. 
Figure 16 and Figure 17 show the trend for the estimated position and velocity. As one can see from Figure 16a and Figure 17a, the position error converges towards low values in less than one day as the first set of measurements are received.

Conversely from Figure $16 \mathrm{~b}$ and Figure $17 \mathrm{~b}$, the estimated velocity error needs between two and four days to converge. The peaks in the velocity error are due to the unavailability of ground station measurements when only on-board sensors are employed. When the Doppler shift is not considered the magnitude of these peaks is higher, with a maximum value of $42.3 \mathrm{~m} / \mathrm{s}$ in case 1 . When the Doppler shift is used, the maximum value slightly reduces to $39.6 \mathrm{~m} / \mathrm{s}$.
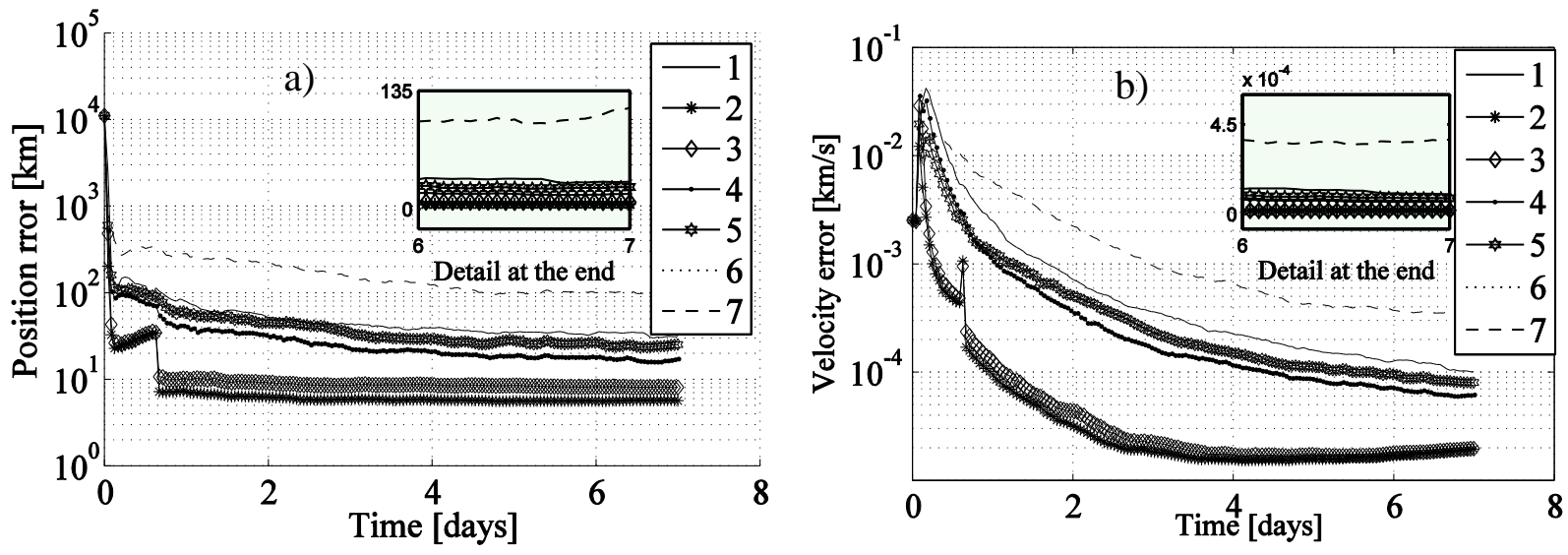

Figure 16. Asteroid estimate a) position and b) velocity error in 7 days combined onboard optical observation measurements with ground station tracking.
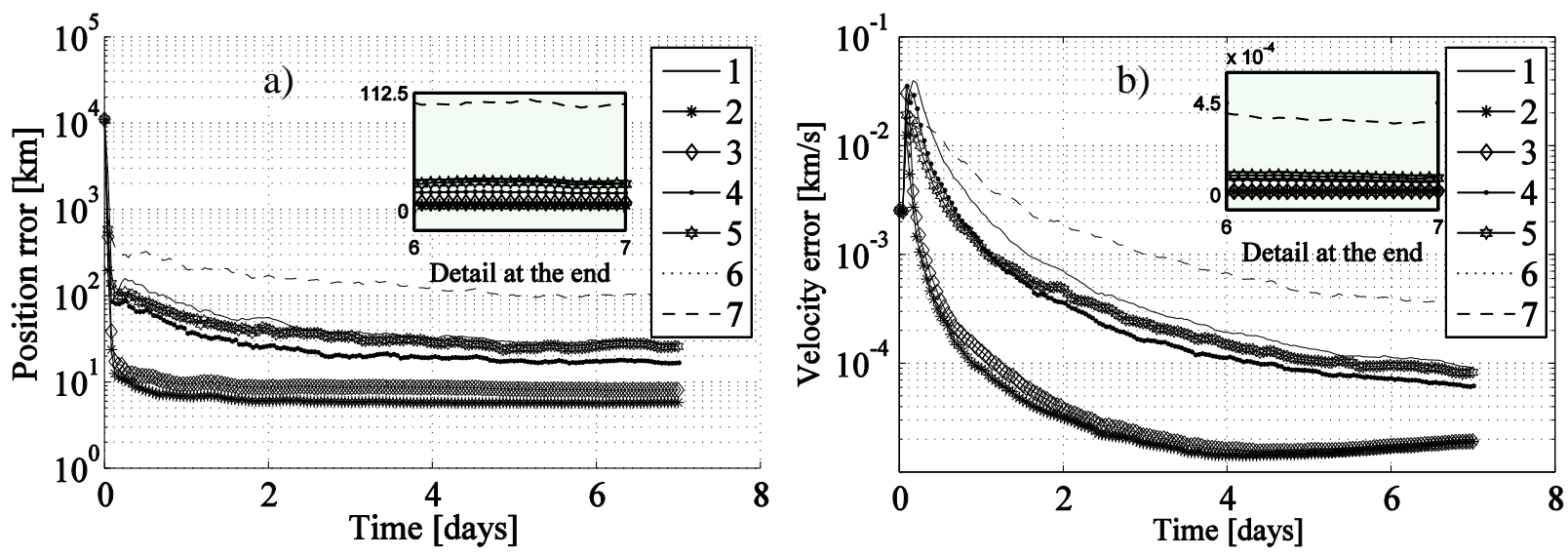

Figure 17. Asteroid estimate a) position and b) velocity error in 7 days combined on-board optical observation measurements with ground station observation and Sun Doppler.

When the two spacecraft are very close as in configuration 7 , the problem is not well conditioned because the measurements from the formation are almost coincident, and, thus, more affected by the measurement error. This can be seen in Figure 16a, where there are small peaks in position till day 4. With Doppler shift measurement, the peaks in position error disappear after 2 days, while the velocity error presents a slightly fastest convergence rate, as shown in Figure 17b.

It can be concluded that the combined use of on-board and ground station measurements improves the position estimate by approximately 3 orders of magnitude in 1 week. The use of Doppler instrument has beneficial effects since it helps the filter converge faster towards lower 
error regions when ground station tracking is not available. Nonetheless, the improvement is not equally significant for all the approach configurations.

\section{Conclusions}

This paper presented different navigation strategies for a 4-spacecraft formation in the proximity of an asteroid. An Unscented $H_{\infty}$ Filter was used to data-fuse the measurements from each spacecraft and estimate their position and velocity with respect to the asteroid Apophis. The choice of the Unscented $H_{\infty}$ Filter emerged from a statistical analysis of the performance of four different filters - EKF, UKF, EHF, UHF - where the EHF and UHF were statistically more accurate. Although the UHF is $20 \%$ more expensive than the EHF and little statistical difference was registered between the accuracy of the EHF and the UHF, the latter was preferred as it is more robust and marginally more accurate when coupled with the control algorithm.

From all the cases presented in this paper, it can be seen that the use of a formation and interspacecraft position measurements improves the navigation accuracy and the robustness against partial or complete failures. Furthermore, it was demonstrated that a formation with a minimum two spacecraft improves the ephemerides of the asteroid by data-fusing the on-board measurements with the tracking of the spacecraft from the ground station.

For the case of 4-spacecraft in close formation, the UHF-based navigation algorithm with collaboration among the members of the formation provided a reduction of the position error by more than $50 \%$, and a reduction of the velocity error by over $60 \%$. The results indicate that the inter-spacecraft measurements aiding navigation can better solve the problem of the orbit determination of spacecraft formation in the proximity of the asteroid. Furthermore, the disaggregated processing of the available measurements significantly improved the resilience of the formation against partial or complete instrument failure.

The combined use of on-board and ground station measurements provided an improvement in the estimated position accuracy by about 3 orders of magnitude while the velocity was as precise as $1 \mathrm{~cm} / \mathrm{s}$. A better knowledge of the asteroid's orbit can be used for correction manoeuvres planning, thus approaching the asteroid could be accomplished with less propellant consumption. The improvement can also be used to refine the trajectories of other potentially hazardous asteroids that the spacecraft formation might encounter during deep space navigation phases.

For future work, an improvement in the dynamics model could include more gravitational harmonics as well as the perturbations due to the asteroid tugging or a deflection action. Finally, the influence of the number of spacecraft on the performance of the navigation system could be considered in view of extending the formation to a swarm.

\section{Acknowledgements}

This work is partially supported by the FP7 Marie Curie ITN, Stardust, Grant Agreement No. 317185.

\section{Appendix}

Figure 18 and Figure 19 report the comparison for SC-1 and SC-2 omitted in Section 6.2. 

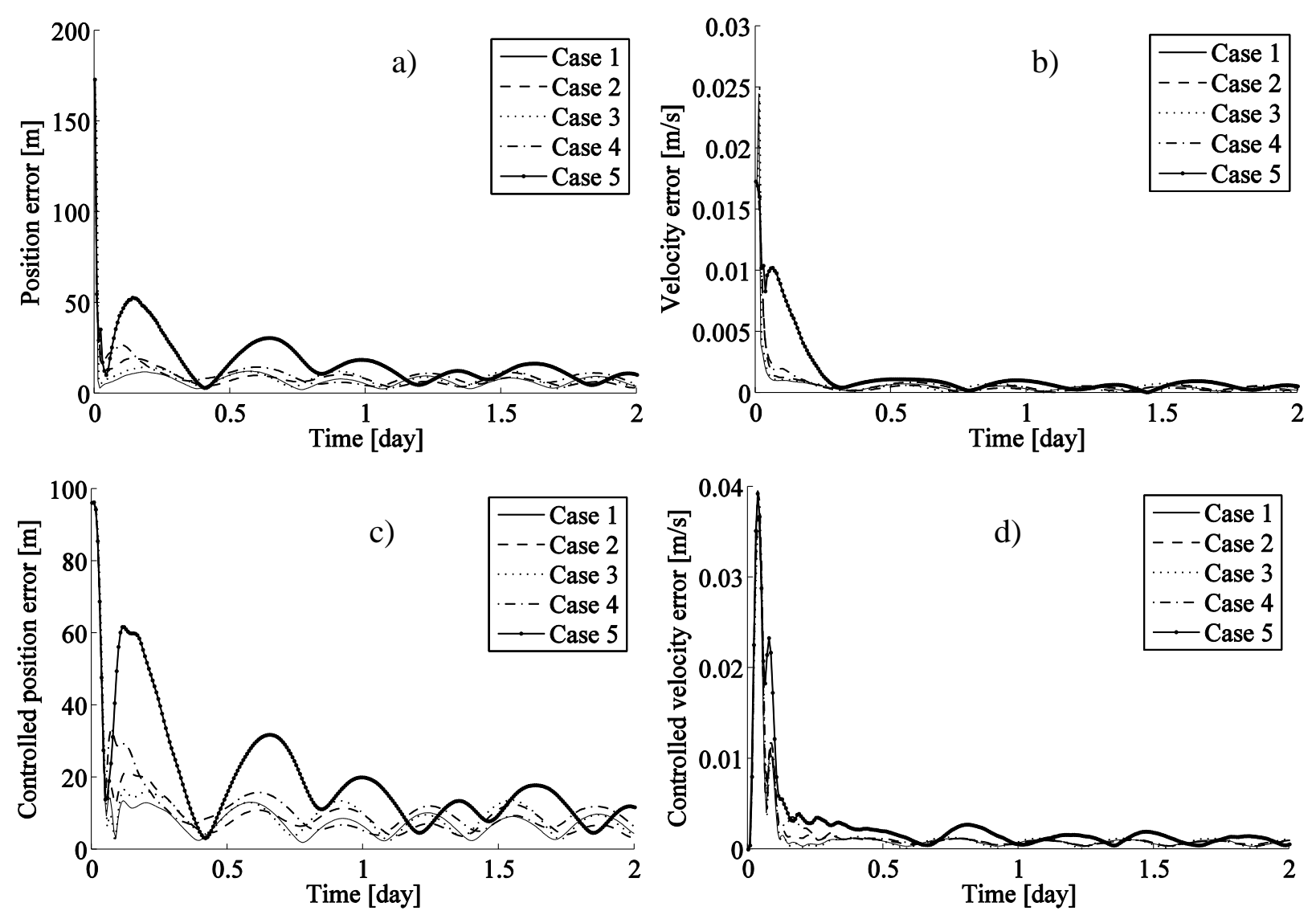

Figure 18. SC-1 failures: RMSE for estimated a) position, b) velocity, c) controlled position and d) controlled velocity

The same conclusions drawn for the other 2 spacecraft can be done. Low levels of measurements error and limited failures (Case 1 to 4 ) do not affect much the performance of the filter and the controller. When the measurement noise is high and many failures occur, as in Case 5, there are oscillations in the estimated and controlled trajectory. Nonetheless this condition is still acceptable since it permits the spacecraft to maintain the formation with the asteroid.
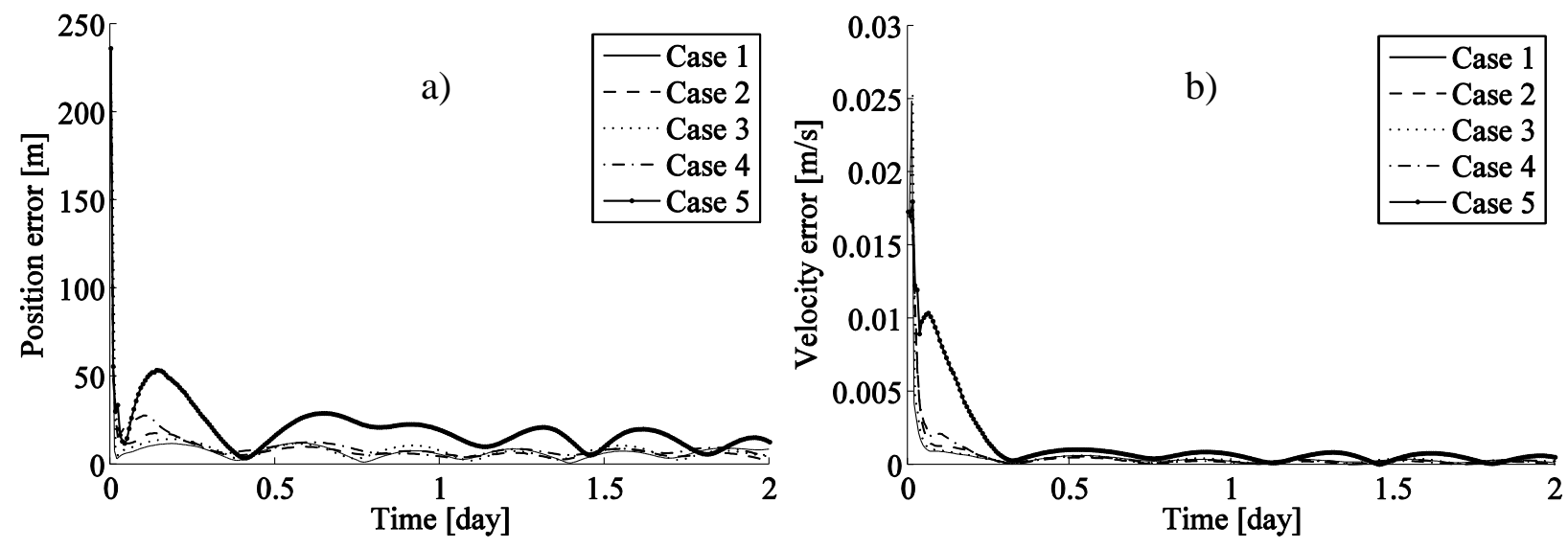

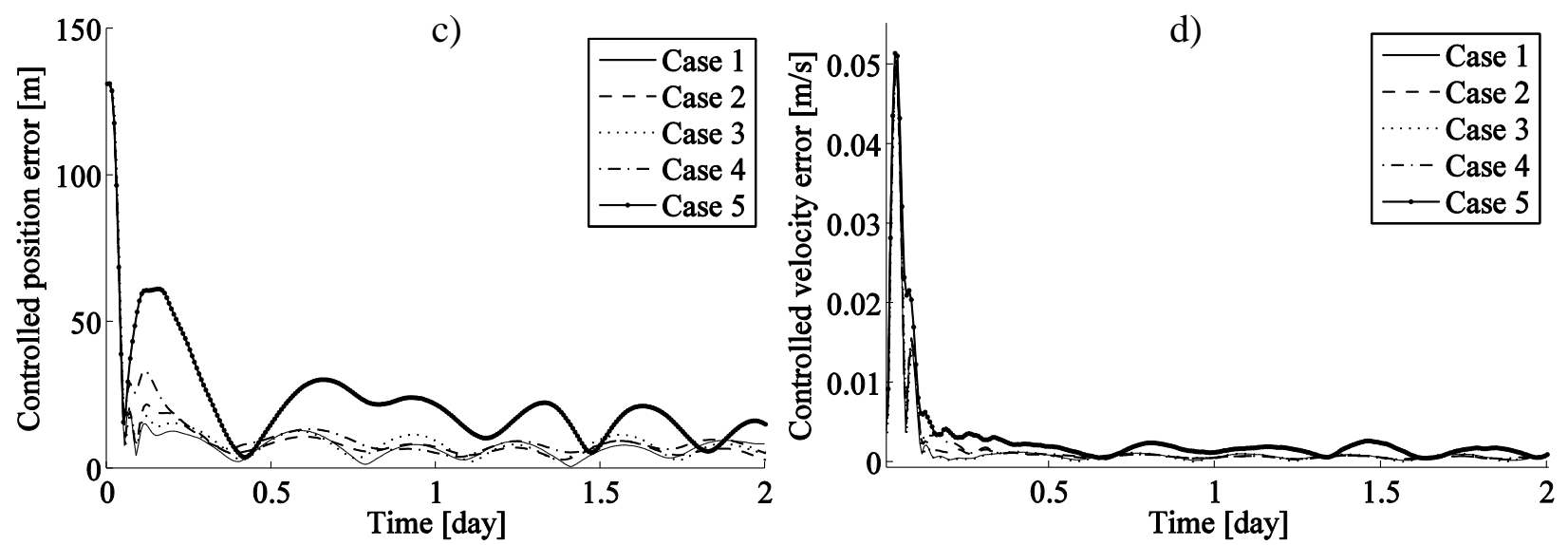

Figure 19. SC-2 failures: RMSE for estimated a) position, b) velocity, c) controlled position and d) controlled velocity

\section{References}

Alonso, R., Du, J., and Hughes, Y Relative Navigation for Formation Flying of Spacecraft. Proceedings of the Flight Mechanics Symposium, NASA-Goddard Space Flight Center, Greenbelt, MD, 2001, pp. 115-129.

Broschart, S. B. and Scheeres, D. J., Control of Hovering Spacecraft Near Small Bodes: Application to Asteroid 35143 Itokawa, Journal of Guidance, Control, and Dynamics 27 (2005) 343-354.

Curtis, S. A., Rilee, M. L., Clark, P. E., Marr G. C. Use of swarm intelligence in spacecraft constellations for the resource exploration of the asteroid belt (2003). Proceedings of the Third International Workshop on Satelllite Constellations and Formation Flying, Pisa, Italy, 24-26, 2003.

Colombo, C., Sanchez Cuartielles, J.P., Vasile, M. et al. A comparative assessment of different deviation strategies for dangerous NEO. International Astronautical Congress. Valencia, Spain, October, 2006.

Colombo, C., Sanchez Cuartielles, J.P., Vasile, M. et al. Comparison of single and multispacecraft configurations for NEA deflection by solar sublimation. In: Belbruno, E. (Ed.), New Trends in Astrodynamics and Applications III. Vol. 886. American Institute of Physics, 2007, pp. 303-316.

Chung, L.R. Orbit determination methods for deep space drag-free controlled laser interferometry missions. Master thesis. University of Maryland, 2006

Cui, P.Y., Chang, X.H. and Cui, H.T. Autonomous orbit determination of deep space probe based on the Sun line-of-sight vector, IEEE, 2010, 540-544.

Crassidis, J.L., and Junkins, J.L. Optimal Estimation of Dynamic Systems, 1st Edition, Chapman \& Hall/CRC Press, Boca Raton, FL, 2004. 
Dionne, K. Improving Autonomous Optical Navigation for Small Body Exploration Using Range Measurements. AIAA 2009-6106. AIAA Guidance, Navigation, and Control Conference, 10 - 13 August 2009, Chicago, Illinois.

Gibbons, J. D. and Chakraborti, S. Nonparametric Statistical Inference, 5th Ed., Boca Raton, FL: Chapman \& Hall/CRC Press, Taylor \& Francis Group, 2011.

Gong, S., Li, J., BaoYin, H. Formation flying solar-sail gravity tractors in displaced orbit for towing near-earth asteroids. Celest. Mech. Dyn. Astron. 105(1), 159-177 (2009).

$\mathrm{Hu}$, W. and Scheeres, D.J. Spacecraft motion about slowly rotating asteroids. Journal of Guidance, Control and Dynamics 25 (4), July-August 2002, 765-775.

Julier, J. K. Uhlmann and Durrant-Whyte, H.F. A new approach for filtering nonlinear systems, Proceedings of the American Control conference, Seattle, Washington, 1995.

Konimato, T., Matsuoka, M., Uo, M., Hashimoto, T. and Kawaguchi, J. Optical Hybrid Navigation in Hayabusa - Approach, Station Keeping \& Hovering, NEC Aerospace Systems, NEC Toshiba Space Systems, ISAS, JAXA, 2006.

Kubotaa, T., Hashimotoa, T., Sawai, S., Kawaguchib, J., Ninomiyaa, K., Uoc, M., Babac, K. An autonomous navigation and guidance system for MUSES-C asteroid landing. Acta Astronautica $52(2003) 125-131$.

Li, S., Cui, P., and Cui, H. Autonomous navigation and guidance for landing on asteroids. Aerospace science and technology, 2006, 10(3): 239-247.

Li, S., Cui, P.Y., Cui, H.T. Vision-aided inertial navigation for pinpoint planetary landing. Aerospace Science and Technology, 2007, 11(6):499-506.

Li, W. and Jia, Y. H-infinity filtering for a class of nonlinear discrete-time systems based on unscented transform. Signal Processing 90(2010):3301-3307.

Long, A., Kelbel, D., Lee, T., Leung, D., Carpenter, J.R., Gramling, C. Relative Navigation Algorithms for Formation-Flying Satellites, ISFF, October 29-31, 2002, Toulouse, France

Maddock, C., Sanchez Cuartielles, J. P., Vasile, M., Radice, G. Comparison of single and multispacecraft configurations for NEA deflection by solar sublimation. In: Belbruno, E. (Ed.), New Trends in Astrodynamics and Applications III. Vol. 886. American Institute of Physics, 2007, pp. 303-316.

Melosh, H.J., Nemchinov, I.V. and Zetzer, Y.I. Non-nuclear strategies for deflecting comets and asteroids. In: Gehrels, T. (Ed.), Hazard due to comets and asteroids. University of Arizona Press, 1994, pp. 1111-1132.

Morley, T. and Budnik, F. Rosetta Navigation for the fly-by of the asteroid 2867 Steins. Proceedings of the $21^{\text {st }}$ International Symposium on Space Flight Dynamics. Toulouse, France, 2009. 
Oh, S.M and Johnson, E. N. Relative Motion Estimation for Vision-based Formation Flight using Unscented Kalman Filter. AIAA Guidance, Navigation and Control Conference and Exhibit, Hilton Head, South Carolina, 2007.

Scheeres, D.J., Gaskell, R., Abe, S., Barnouin-Jha, O., Hashimoto, T., Kawaguchi, J., Kubota, T., Saito, J., Yoshikawa, M., Hirata, N., Mukaik, T., Ishiguro, M., Kominato, T., Shirakawa, K. and Uo, M. The Actual Dynamical Environment About Itokawa. AIAA/AAS Astrodynamics Specialist Conference and Exhibit. 16 - 19 August 2004a, Keystone, Colorado.

Scheeres, D.J., Broschart, S., Ostro, S.J. and Benner, L.A. The Dynamical Environment About Asteroid 25143 Itokawa: Target of the Hayabusa Mission. AIAA/AAS Astrodynamics Specialist Conference and Exhibit. 16 - 19 August 2004b, Providence, Rhode Island.

Scheeres, D.J. Interactions between ground-based and autonomous navigation for precision landing at small solar-system bodies. The Telecommunications and Data Acquisition Progress Report 42-132, October-December 1998, pp: 1-12.

Scheeres, D.J. Orbit Mechanics about Asteroids and Comets. Journal of Guidance, Control and Dynamics, 2012, 35(3): 987-997.

Shim, S., Park, S.Y., and Choi, K.H. Autonomous Real-time Relative Navigation for Formation Flying Satellites. Journal of Astronomy and Space Sciences, 2009, 26: 59-74.

Simons, D. Optimal state estimation, Kalman, $H_{\infty}$ and Non-linear Approaches. Published by John Wiley \& Sons, Inc., Hoboken, New Jersey, 2006.

Thornton, C. L. and Border, J.S. Radiometric Tracking Techniques for Deep-Space Navigation, JPL Deep Space Communications and Navigation Series, John Wiley \& Sons Inc., Hoboken, New Jersey, 2003.

Turconi, A., Palmer, P.L., Roberts, M. Autonomous guidance and control in the proximity of asteroids using a simple model of the gravitational potential. IAA-AAS-DyCoSS2-14 -02-01, 2nd IAA Conference on Dynamics and Control of Space Systems, March 24-26, 2014, Rome, Italy.

Vasile, M. and Maddock, C.A. Design of a Formation of Solar Pumped Lasers for Asteroid Deflection. Advances in Space Research, 2012, 50(7): 891-905.

Vetrisano, M. and Vasile, M. Collaborative guidance navigation and control of disaggregated spacecraft in the proximity of minor bodies. In: $63^{\text {rd }}$ International Astronautical Congress, Naples, Italy, 2012.

Vetrisano, M., Branco, J. and Garcia-Yarnoz, D. Effective approach navigation prior to small body deflection. 64th International Astronautical Congress, Beijing, China, 2013.

Wie, B.. Dynamics and control of gravity tractor spacecraft for asteroid deflection. Journal of Guidance, Control, and Dynamics, 2008, 31(5): 1413-1423.

Winkler, T., Kaplingera B., Wie, B. Optical Navigation and Fuel-Efficient Orbit Control Around 
an Irregular-Shaped Asteroid. IAA-PDC13-04-28, Planetary Defense Conference 2013, April 1519, 2013, Flagstaff, Arizona, USA.

Yim, J.R., Crassidis, J.L. and Junkins, J.L. Autonomous Orbit Navigation of Interplanetary Spacecraft. AIAA Guidance, 2000. 